\title{
TOG-tubulin binding specificity promotes microtubule dynamics and mitotic spindle formation
}

\author{
Amy E. Byrnes ${ }^{1,2}$ and Kevin C. Slep ${ }^{2,3}$ \\ 'Department of Biochemistry and Biophysics, 2Program in Molecular and Cellular Biophysics, and 3epartment of Biology, University of North Carolina, Chapel Hill, NC \\ 27599
}

XMAP215, CLASP, and Crescerin use arrayed tubulin-binding tumor overexpressed gene (TOG) domains to modulate microtubule dynamics. We hypothesized that TOGs have distinct architectures and tubulin-binding properties that underlie each family's ability to promote microtubule polymerization or pause. As a model, we investigated the pentameric TOG array of a Drosophila melanogaster XMAP215 member, Msps. We found that Msps TOGs have distinct architectures that bind either free or polymerized tubulin, and that a polarized array drives microtubule polymerization. An engineered TOG 1-2-5 array fully supported Msps-dependent microtubule polymerase activity. Requisite for this activity was a TOG5-specific $\mathrm{N}$-terminal HEAT repeat that engaged microtubule lattice-incorporated tubulin. TOG5-microtubule binding maintained mitotic spindle formation as deleting or mutating TOG5 compromised spindle architecture and increased the mitotic index. Mad2 knockdown released the spindle assembly checkpoint triggered when TOG5-microtubule binding was compromised, indicating that TOG5 is essential for spindle function. Our results reveal a TOG5-specific role in mitotic fidelity and support our hypothesis that architecturally distinct TOGs arranged in a sequence-specific order underlie TOG array microtubule regulator activity.

\section{Introduction}

Microtubules (MTs) are dynamic polymers of $\alpha \beta$-tubulin that transition between phases of polymerization, pause, and depolymerization (Mitchison and Kirschner, 1984). Spatial and temporal control of these phases is a key requirement for MT-dependent cellular restructuring events including mitosis, vesicle trafficking, ciliogenesis, and cell migration. MTassociated proteins (MAPs) regulate MT dynamics. Several MAPs, including members of the XMAP215, Crescerin, and CLASP families, are composed of arrayed tubulin-binding tumor overexpressed gene (TOG) domains (Akhmanova et al., 2001; Cassimeris et al., 2001; Leano et al., 2013; Das et al., 2015). Interestingly, these three families use their TOG arrays to differentially affect MT dynamics: XMAP215 promotes MT polymerization in interphase and mitosis, Crescerin promotes proper MT architecture in the primary cilium, and CLA SP promotes MT pause/rescue (Gard and Kirschner, 1987; Tournebize et al., 2000; Akhmanova et al., 2001; Bratman and Chang, 2007; Al-Bassam et al., 2010; Das et al., 2015). How these three families use a common domain to differentially affect MT dynamics and organization is poorly understood. To date, several TOG domain structures from each family have been determined and are beginning to reveal distinct TOG domain architectures along each array. From these results, we hypothesized that specific arrays of TOG domains with dis-

Correspondence to Kevin C. Slep: kslep@bio.unc.edu

Abbreviations used: CD, circular dichroism; Ce, Caenorhabditis elegans; CTD, C-terminal domain; FL, full-length; HR, HEAT repeat; MAP, microtubuleassociated protein; MT, microtubule; rmsd, root-mean-square deviation; SAC, spindle assembly checkpoint; TOG, tumor overexpressed gene. tinct architectures and concomitant tubulin-binding properties underlie each family's unique regulatory effect on MT dynamics. To test this hypothesis, we investigated the pentameric TOG array of the Drosophila melanogaster XMAP215 MT polymerase family member Msps.

XMAP215 family members promote rapid processive MT polymerization and drive bipolar spindle formation using an N-terminal TOG array (Gard and Kirschner, 1987; Vasquez et al., 1994; Cullen et al., 1999; Popov et al., 2001; Brouhard et al., 2008; Widlund et al., 2011). TOG domains bind tubulin using an $\alpha$-solenoid structure formed by six HEAT repeats (HRs; Al-Bassam et al., 2007; Slep and Vale, 2007). The number of TOG domains across XMAP215 family members varies. Yeast XMAP215 members homodimerize, giving them a total of four functional TOG domains, whereas non-yeast XMAP215 members contain a pentameric TOG array and function as monomers (Cassimeris et al., 2001; De Wulf et al., 2003; van Breugel et al., 2003; Al-Bassam et al., 2006). Caenorhabditis elegans $(\mathrm{Ce}$ ) ZYG-9 is a notable exception, containing a trimeric array (Matthews et al., 1998; Al-Bassam et al., 2007). Why the number of TOG domains across XMAP215 family members varies is unknown, but it suggests differential TOG domain function as well as the evolution of honed, minimal, polymerization-competent TOG arrays for yeast and $\mathrm{Ce}$. Evi-

(C) 2017 Byrnes and Slep This article is distributed under the terms of an AttributionNoncommercial-Share Alike-No Mirror Sites license for the first six months after the publication date (see http://www.rupress.org/terms/). After six months it is available under a Creative Commons License (Attribution-Noncommercial-Share Alike 4.0 International license, as described at https://creativecommons.org/licenses/by-nc-sa/4.0/). 
dence supporting differential TOG function comes from studies that mutated TOG tubulin-binding activity in individual, paired, and arrayed TOG domains, which differentially affected XMAP215 activity. Mutations that ablated TOG1 or TOG2 tubulin-binding activity severely compromised MT polymerization rates and spindle size, whereas mutations in TOGs 3 and 4 had less dramatic effects (Widlund et al., 2011; Al-Bassam et al., 2012; Reber et al., 2013; Fox et al., 2014). To date, mutating residues in TOG5 does not appreciably affect MT polymerization or mitotic spindle size (Widlund et al., 2011; Reber et al., 2013; Fox et al., 2014). This raises the question, what is the molecular basis for the differential roles TOG domains play in the XMAP215 MT polymerization mechanism?

In parallel with the differential effects observed when the tubulin-binding activities of specific TOG domains were mutated, TOG structures determined to date reveal distinct architectures along the array. TOG1 and TOG2 have similar architectures that complement the bent architecture of free, unpolymerized tubulin (Slep and Vale, 2007; Ayaz et al., 2012, 2014). TOG3 is structurally distinct from TOGs 1 and 2 but has a tubulin-binding surface similar to TOG1 and TOG2 (Howard et al., 2015). The structure of TOG4 is dramatically distinct and has a unique bend across its three $\mathrm{C}$-terminal HRs that predicts a novel tubulin-binding mode (Fox et al., 2014). No structure of TOG5 from a pentameric TOG array has been reported to date. Based on primary sequence analysis, it was hypothesized that TOG5 is similar to the last TOG domain in Ce ZYG-9, TOG3 (Al-Bassam et al., 2007; Al-Bassam and Chang, 2011). Previous work determined the structure of ZYG-9 TOG3 and revealed a unique, extra, N-terminal HR positioned orthogonal to the six HRs in the $\alpha$-solenoid (Al-Bassam et al., 2007). The importance of this extra HR, whether it plays a role in TOG-tubulin binding, and whether ZYG-9 TOG3 is equivalent to TOG5 from pentameric XMAP215 family arrays are still unclear. Of the five TOG domains in the pentameric array, TOG5 is the most divergent and enigmatic of the TOG domains. The sequence homology between TOG5 and ZYG-9 TOG3 strongly suggests a conserved role for this TOG domain in XMAP215 function, but whether this role involves MT polymerase activity or is embedded in the complex functions XMAP215 plays during mitosis remains unknown.

Here, we investigate the Msps pentameric TOG array in interphase and mitosis. We show that, along with the Cterminal domain (CTD), TOGs 1,2 , and 5 are necessary and collectively sufficient to fully rescue interphase MT polymerization rates. We report the structure of Msps TOG5, revealing a conserved $\mathrm{N}$-terminal $\mathrm{HR}$ that binds polymerized tubulin and engages lateral $\alpha \beta$-tubulin heterodimers on the MT lattice, which is necessary for full Msps-mediated MT polymerization activity and mitotic spindle formation. TOG5 MT-binding activity contrasts with the activities of TOGs 1 and 2 that specifically bind free tubulin. Shuffling the order of TOG domains that bind either free or MT lattice-incorporated tubulin reduces MT polymerization rates. Collectively, this confirms our hypothesis that distinct TOG domain architectures along an array evolved to bind specific tubulin structural states and that XMAP215 activity requires a polarized TOG array. Our findings support a larger TOG array paradigm applicable to the XMAP215, Crescerin, and CLASP families in which structurally distinct TOG domains with unique tubulinbinding architectures arranged in a specific order are used to differentially regulate MT dynamics.

\section{Results}

TOG domains 1,2 , and 5 constitute a minimal TOG domain array that rescues MT polymerization rates

Unlike yeast and Ce ZYG-9, most eukaryotic XMAP215 family members contain pentameric TOG arrays. To test whether all five TOGs are necessary to facilitate MT polymerization, we systematically deleted Msps TOG domains and performed MT polymerization rescue experiments in Drosophila S2 cells (Fig. 1 A). We treated S2 cells with msps dsRNA to deplete endogenous Msps and used EB1 comet velocity as a readout for MT polymerization rates (Fig. 1 B). Msps depletion reduced MT polymerization rates from 12.3 to $5.5 \mu \mathrm{m} / \mathrm{min}$, which was rescued by transient transfection of full-length (FL) Msps (Fig. 1, C-F). As expected, removing all five TOG domains from the array prevented rescue of MT polymerization velocity. TOG5 deletion partially impaired MT polymerization, yielding a rate of $9.8 \mu \mathrm{m} / \mathrm{min}$ (Fig. $1 \mathrm{G}$ ). To mimic the TOG domain architecture of ZYG-9, we created two constructs lacking TOGs 3 and 4 . Both of these constructs fully rescued MT polymerization rates, indicating that TOG domains 1,2 , and 5 are sufficient to drive Msps-mediated MT polymerization. To further test the necessity of these domains, we created $\Delta 12, \Delta 345$, and $\Delta 45$ constructs. Removing TOG domains 1 and 2 from the array fully abrogated MT polymerization rescue activity (Fig. $1 \mathrm{G}$ ). In contrast, $\Delta 345$ and $\Delta 45$ constructs impaired MT polymerization to rates on par with the $\Delta 5$ construct (Fig. $1 \mathrm{G}$ ). We note that there is no correlation between Msps-eGFP construct expression levels and MT polymerization velocity for the cells examined in this study (Fig. S1). These data indicate that TOG domains 1, 2, and 5 constitute a minimal TOG domain array that can fully rescue MT polymerization rates. This supports the hypothesis that TOG5 is positionally equivalent to ZYG-9 TOG3 and is consistent with sequence alignments showing high conservation between these two domains (Fig. S2 A). Furthermore, these data suggest an evolutionarily conserved role for TOG5 in the XMAP215 polymerization mechanism.

Msps TOG5, like ZYG-9 TOG3, has an additional $\mathbf{N}$-terminal $\mathbf{H R}$ containing conserved tubulin-binding residues

To test whether TOG5 is structurally similar to ZYG-9 TOG3, we determined the crystal structure of Drosophila Msps TOG5 (Fig. 2 A). We obtained crystals in the space group P $2{ }_{1} 2{ }_{1} 2_{1}$ and solved the structure using ZYG-9 TOG3 as a molecular replacement model. Final iterative refinement yielded $\mathrm{R}$ and $\mathrm{R}_{\text {free }}$ values of 0.19 and 0.22 , respectively (Table 1 ). The model encompasses residues $1,145-1,406$ and contains two molecules in the asymmetric unit. TOG5 includes a core body of six HRs, labeled A-F (Fig. 2 A). Unlike TOGs 1-4, however, TOG5 has an additional N-terminal HR (HR 0, red) that is positioned orthogonal to HRs A-F. Other TOG domains contain architectural features outside the TOG domain body that promote TOG domain structural stability (Das et al., 2015; Howard et al., 2015). To test whether TOG5 HR 0 maintains TOG5 stability, we created a series of point mutations at the interface between HR 0 and HRs A-C (Fig. S2, B-D). Mutating R1157 to glutamic acid destabilized TOG5, preventing purification of this protein (Fig. S2 B). Recombinant protein was purified for W1169E/ E1161R mutants, and circular dichroism (CD) analyses showed that mutating these residues along the binding interface does 
A
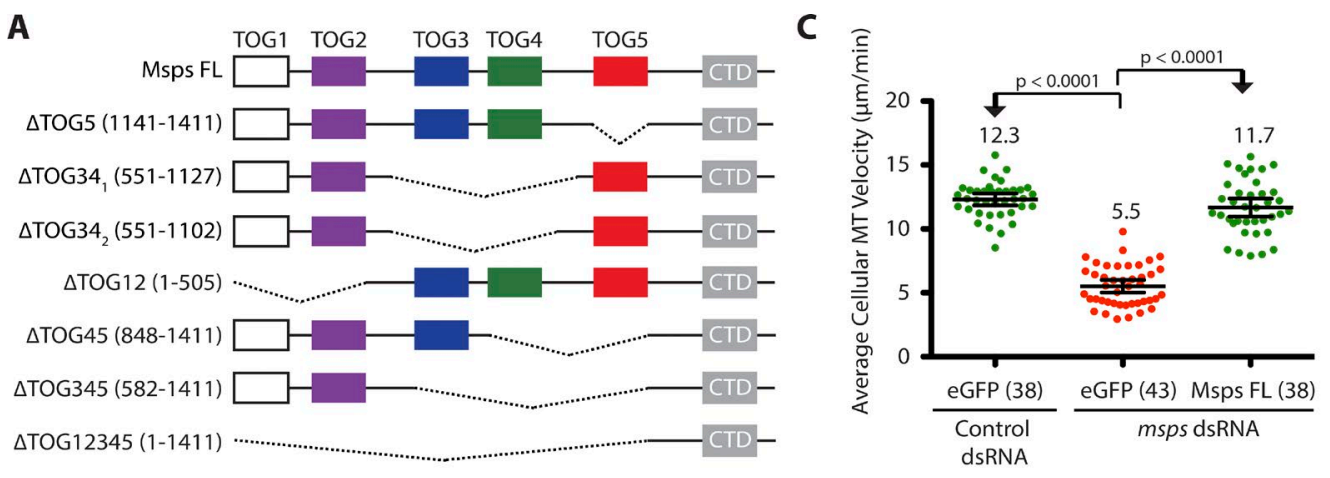

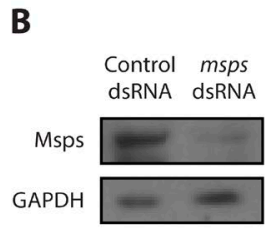

E

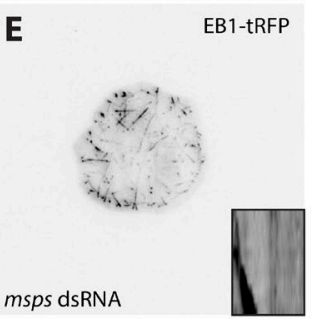

D

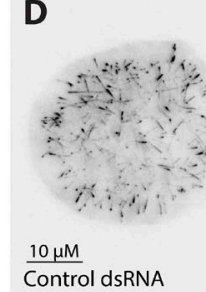

$\mathbf{F}$

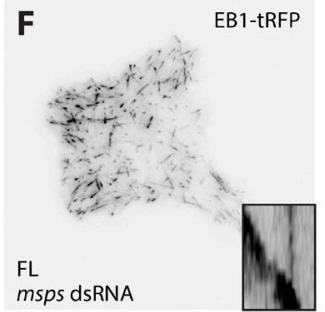

EB1-tRFP
G

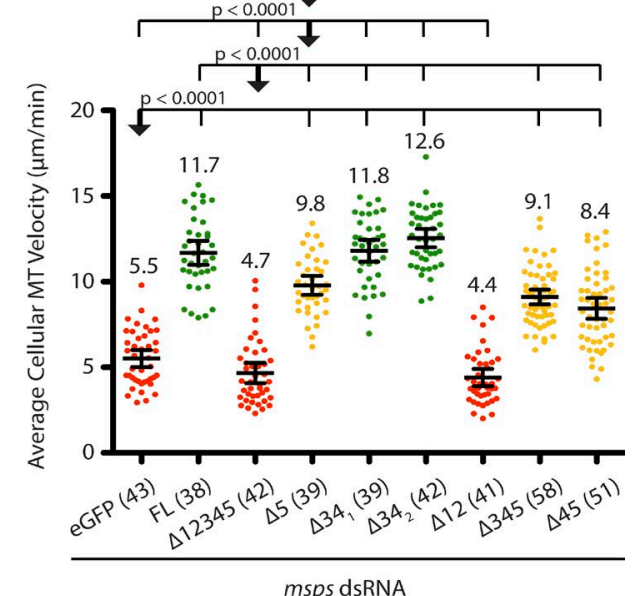

Figure 1. TOG domains 1, 2, and 5 are sufficient to rescue MT polymerization rates. (A) Msps-eGFP TOG domain deletion constructs examined in S2 cells for their ability to rescue MT polymerization rates using EB 1-tRFP as a readout (C-G). (B) Treating S2 cells with msps 5' and 3' UTR-directed dsRNA reduces endogenous Msps by $>80 \%$ (Western blot). (C) EB1 comet velocity is drastically reduced in msps dsRNA-treated S2 cells, but rescue is obtained upon FL Msps transfection. (D-F) Representative images of control (D) or msps dsRNA (E) and FL rescue (F) S2 cells and a corresponding EBl comet kymograph. (G) EBI comet velocities from TOG domain deletion experiments. Removing all five TOG domains or TOGs 1 and 2 eliminates rescue activity. Deleting TOGs 3 and 4 does not affect rescue; however, $\Delta 5$ significantly reduces MT polymerization rates, which is mirrored with Msps $\Delta 345$ and $\Delta 45$ constructs. Number of cells analyzed is shown in parentheses. Mean $\pm 95 \%$ confidence intervals are shown; two-tailed Mann-Whitney $U$ test.

not change the $\alpha$-helical character of TOG5, but does reduce TOG5 stability (E1161R; Fig. S2, C-E; and Fig. S4, A-C). To probe architectural conservation, we superimposed Msps TOG5 with ZYG-9 TOG3 (Al-Bassam et al., 2007). These domains both contain HR 0 and align well, with a root-mean-square deviation of $1.4 \AA$ (Fig. 2 B). The structure of Msps TOG5 confirms our hypothesis that the last TOG domain from pentameric XMAP215 family members is structurally equivalent to the last TOG domain in the ZYG-9 trimeric TOG array. The conserved nature of TOG5 and ZYG-9 TOG3 suggests that these domains play a core mechanistic role in XMAP215-mediated MT regulation and confirms that the Msps TOG1-2-5 construct functionally and structurally mimics the ZYG-9 trimeric TOG array.

Recent Stu2 TOG1- and TOG2- $\alpha \beta$-tubulin complex structures revealed that these TOG domains each bind a single $\alpha \beta$-tubulin heterodimer using conserved intra-HEAT loop residues spanning one face of each domain (Ayaz et al., 2012, 2014). We examined whether TOG5 shared this tubulin-binding surface by investigating TOG5 residue conservation and electrostatics. TOG5's intra-HEAT loops are conserved across the XMAP215 family and have a standard basic character that could complement the acidic surface of tubulin (Fig. 2, C and D; Baker et al., 2001). We next assessed whether TOG5 could make contacts with tubulin similar to those observed with
TOGs 1 and 2 by aligning TOG5 to the Stu2 TOG1- $\alpha \beta$-tubulin complex structure (Fig. 2 E; Ayaz et al., 2012). When modeled on the Stu2- $\alpha \beta$-tubulin complex structure, conserved TOG5 determinants are positioned to make canonical TOG-tubulin interactions (Fig. 2 F). In TOG-tubulin structures determined to date, a key conserved HR A loop tryptophan is used to engage $\beta$-tubulin. In contrast to the HR A loop tryptophan present in TOGs $1-4$, TOG5 contains a conserved phenylalanine (F1204) in this position. Other conserved TOG5 residues flanking F1204 include R1205 and N1244. All of these residues are positioned similarly to the homologous Stu2 TOG1 tubulin-binding residues W23, K24, and N68 (Fig. 2 F). These results support the hypothesis that TOG5 engages tubulin using canonical TOGtubulin binding determinants.

\section{TOG5 HR 0 is positioned to engage laterally associated tubulin heterodimers on the MT lattice}

Tubulin exists in a curved state in solution and undergoes a curved-to-straight transition upon polymerization (Nogales et al., 1998; Rice et al., 2008; Brouhard and Rice, 2014). To examine whether TOG domains could be honed to bind different forms of tubulin, we used the Stu2 TOG1- $\alpha \beta$-tubulin structure as a guide to superimpose different TOG domains onto free 
A

A Tubulin Binding Face

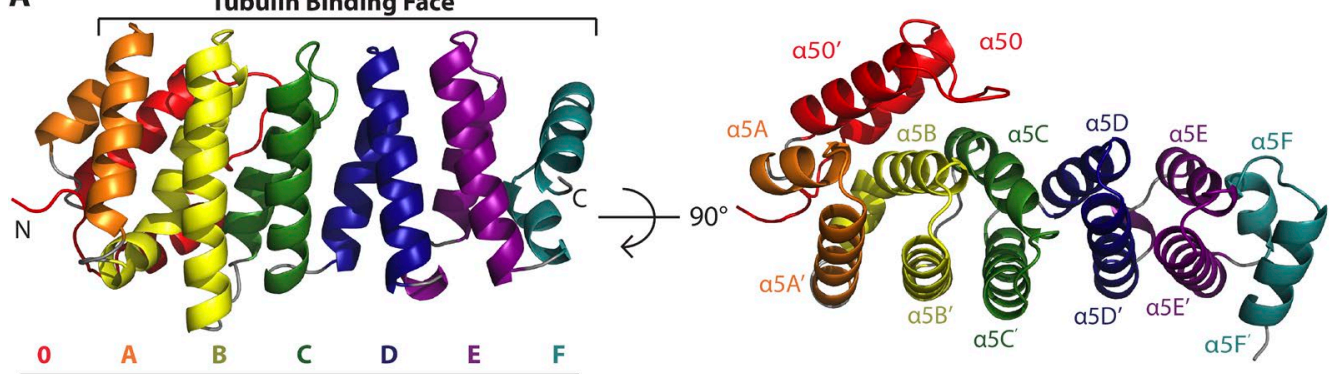

HEAT Repeat (HR)

B

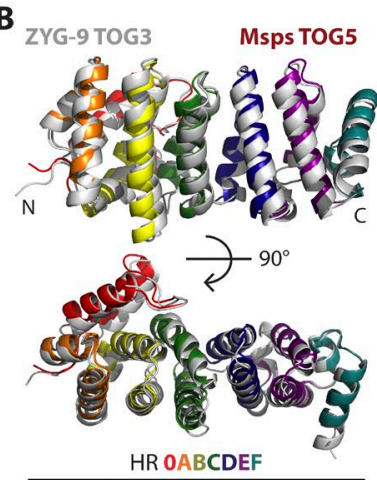

$1.4 \AA ̊$ rmsd
C

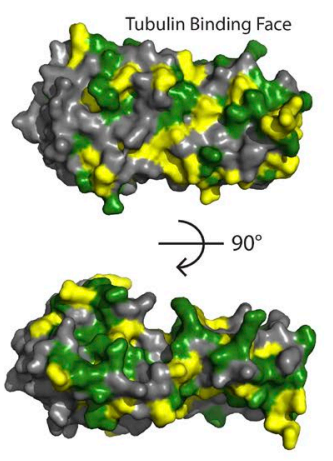

80\% Identity $\quad 80 \%$ Similarity

Residue Conservation

D

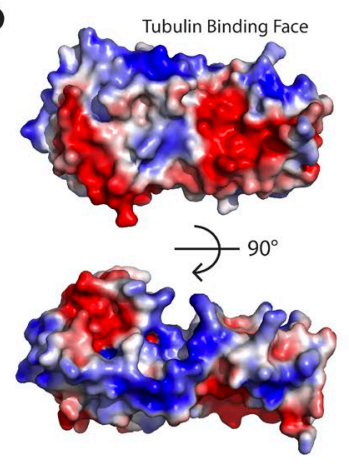

$-3.0 k_{B} T / e \square+3.0 k_{B} T / e$

Electrostatic Potential

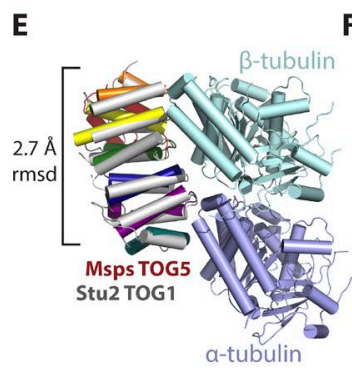

$\mathbf{F}$
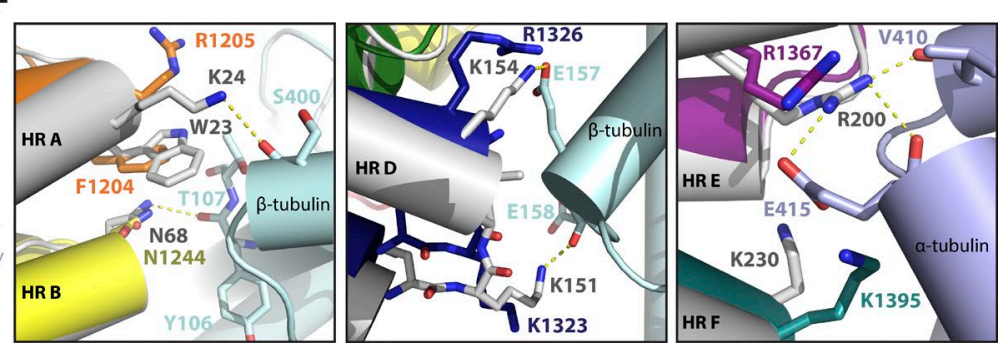

Figure 2. A conserved N-terminal HR (HR 0) distinguishes Msps TOG5 from TOG domains 1-4. (A) Msps TOG5 is a canonical TOG domain, consisting of six HRs (A-F). However, TOG5 has a unique conserved feature, an additional N-terminal HR (HR 0, red), which distinguishes TOG5 from TOGs 1-4. Right, view of the tubulin-binding face. (B) Drosophila Msps TOG5 and Ce ZYG-9 TOG3 both contain HR O and align well, with a root-mean-square deviation of $1.4 \AA$. (C) TOG5 residue conservation based on the multispecies alignment presented in Fig. S2 and oriented as in A and B. (D) Electrostatic potential mapped on TOG5 and oriented as in A-C. (E) Stu2 TOG1 (gray) and Msps TOG5 (color) align with a root-mean-square deviation of $2.7 \AA$ when superimposed on the Stu2 TOG $1 \alpha \beta$-tubulin structure (4FFB). (F) Msps TOG5 is predicted to engage $\alpha \beta$-tubulin across HRs A-F using intra-HEAT loop determinants similar to those in Stu2 TOG1. Msps TOG5 residues shown in color, aligned to the Stu2 (gray)-tubulin complex as in E.

and polymerized tubulin. Stu2 TOG1 (4FFB) and Msps TOG5 (modeled) contact curved $\alpha \beta$-tubulin across their tubulin-binding surface (Fig. 3, A and B). When modeled on straight tubulin using $\beta$-tubulin as a reference, TOG1 becomes disengaged from $\alpha$-tubulin (Fig. 3 A). In contrast, TOG5 maintains contact with $\alpha$-tubulin (Fig. 3 B), suggesting that TOG5 may preferentially bind straight tubulin. To further investigate potential TOG-MT binding modes, we aligned the Stu 2 TOG1- $\alpha \beta$-tubulin structure to the MT lattice coordinates from the Mal3-GTP $\gamma$ S MT EM structure and used this composite model to align other TOGMT interactions (Ayaz et al., 2012; Maurer et al., 2012). When modeled on the MT lattice, Stu2 TOG1 does not engage lateral $\alpha \beta$-tubulin subunits on neighboring protofilaments (Fig. $3 \mathrm{C}$ ). In stark contrast, modeling predicts that TOG4 uses its conserved bend across HRs D-F and TOG5 uses its distinct HR 0 feature to engage laterally associated tubulin. Collectively, these models suggest that TOG domains use distinct architectures to bind free, pseudopolymerized, or lattice-incorporated $\alpha \beta$-tubulin. We note that although the MT-binding footprints modeled for TOG4 and TOG5 are distinct, they overlap with the binding sites for other MAPs including Mal3 (Maurer et al., 2012) and doublecortin (Liu et al., 2012; Fig. S3), suggesting mutually exclusive modes of regional MT plus end binding.

\section{HR O contributes to TOG5 MT lattice} binding, nucleation, and polymerization To further probe whether TOG5 uses its unique architecture to bind the MT lattice and promote Msps polymerase activity, we created a series of point mutations in TOG5 HRs 0-A that we predicted would ablate lateral MT lattice interactions and compared the activity of these mutants to wild-type TOG5 constructs in a number of MT binding/dynamics assays (Fig. 4, A-D; and Fig. 5, A-F). We targeted three TOG5 residues for mutation: K1193, R1175, and M1164. K1193, like Mal3 K63, is positioned to interact with $\alpha$-tubulin on an adjacent tubulin heterodimer (Fig. 4 B). Previous studies showed that a Mal3 
Table 1. Data collection and refinement statistics

\begin{tabular}{|c|c|}
\hline Attribute & Msps TOG5 $(1,141-1,411)$ \\
\hline PDB code & $5 \mathrm{VJC}$ \\
\hline \multicolumn{2}{|l|}{ Data collection } \\
\hline Beamline & GM/CA-CAT 23-IDB \\
\hline Wavelength & 1.03319 \\
\hline Space group & $\mathrm{P} 2{ }_{1} 2_{1} 2_{1}$ \\
\hline Resolution $(\AA)$ & $29.3-2.00(2.07-2.00)$ \\
\hline Unit cell dimensions: $a, b, c(\AA)$ & $53.3,80.0,146.8$ \\
\hline Angles $\alpha, \beta, \gamma\left({ }^{\circ}\right)$ & $90.0,90.0,90.0$ \\
\hline Reflections: measured/unique ( $n)$ & $85,812(8,462) / 43,212(4,251)$ \\
\hline Multiplicity & $2.0(2.0)$ \\
\hline $\mathrm{CCl} 1 / 2$ & $0.992(0.730)$ \\
\hline Completeness (\%) & $95.6(86.0)$ \\
\hline $\mathrm{I} / \sigma$ & $17.7(2.0)$ \\
\hline$R_{\text {merge }}$ & $0.028(0.401)$ \\
\hline \multicolumn{2}{|l|}{ Refinement } \\
\hline Resolution $(\AA)$ & $29.3-2.00(2.07-2.00)$ \\
\hline$R / R_{\text {free }}(\%)^{\text {ab }}$ & $18.7(27.2) / 22.2$ (35.0) \\
\hline Protein/solvent atoms ( $n)$ & $4,110 / 276$ \\
\hline rmsd bond angles $\left({ }^{\circ}\right)$ & 0.008 \\
\hline rmsd bond lengths $(\AA)$ & 0.999 \\
\hline Ramachandran favored/allowed (\%) & $99.21 / 0.79$ \\
\hline Mean $B\left(\AA^{2}\right)$ & 31.3 \\
\hline Phase error $\left({ }^{\circ}\right)$ & 23.0 \\
\hline
\end{tabular}

Values in parentheses are for the highest-resolution shells unless otherwise denoted rmsd, root-mean-square deviation.

a $R=\sum\left(\left|F_{\text {obs }}\right|-k\left|F_{\text {calc }}\right|\right) / \Sigma\left|F_{\text {obs }}\right|$.

${ }^{\mathrm{b}} R_{\text {free }}$ was calculated using a $4.5 \%$ subset of the data that is removed randomly from the original data and excluded from refinement.

K63D mutation eliminated Mal3-MT binding (Maurer et al., 2012). The positional similarity with TOG5 K1193 suggests that this feature may be a conserved MAP-MT interaction determinant, so we generated a K1193E construct. We also mutated HR 0 R1175 and M1164, which interact with lateral $\beta$-tubulin in our docked model (Fig. 4, C and D). We mutated these residues to glutamate to introduce a potential repulsive charge to compromise binding to lateral tubulin subunits on the MT lattice. Mutating single or multiple predicted TOG5-MT binding residues does not affect TOG5 domain architecture or stability as measured by $\mathrm{CD}$, providing license to use these mutations to analyze TOG5-MT binding (Fig. S4, B-I).

We performed both in vitro MT co-sedimentation and in vivo cellular MT localization experiments using a library of GST-dimerized Msps Linker4-TOG5 (1,085-1,411) constructs to examine TOG5-MT binding (Figs. 5 A and S4 A). GST Linker4-TOG5 bound and co-sedimented with taxol-stabilized MTs. However, when predicted lateral $\alpha \beta$-tubulin binding residues (K1193, R1175, or M1164) were individually mutated, MT binding was decreased (Fig. 5 B). These results were mirrored in in vivo cellular MT localization assays. Single TOG5 point mutants decreased GST Linker4-TOG5 localization to the MT lattice. MT lattice binding was further reduced when multiple lateral $\alpha \beta$-tubulin binding and HR A mutations were introduced (Fig. 5, C and D; the HR $\mathrm{A}^{\mathrm{F} 1204 \mathrm{E}}$ mutation is a standard TOG mutation that ablates HR A- $\beta$-tubulin binding; Slep and Vale, 2007). TOG5's MT-binding activity contrasts with the architectural specificity of TOGs 1 and 2 that preferentially bind free tubulin. Because TOG5 HRs 0-A promoted MT-lattice binding activity, potentially by engaging laterally associated tubulin, we hypothesized that TOG5 could potentiate tubulin- tubulin interactions and thus promote MT nucleation. We tested this hypothesis using a light scattering assay (Fig. 5 E). Tubulin alone polymerized at $37^{\circ} \mathrm{C}$ after a lag time of $\sim 500 \mathrm{~s}$. When TOG 1 or 2 was incubated with tubulin, MT polymerization lag time was increased, potentially because of a sequestration effect of these domains binding free tubulin heterodimers (Fig. 5 E). Our light scattering assay also showed that TOG3, which was recently described to have a TOG-tubulin binding architecture similar to TOGs 1 and 2 (Howard et al., 2015), also increased the MT polymerization lag time (Fig. 5 E). In dramatic contrast, TOG domains 4 and 5 instead promoted MT nucleation within the first $100 \mathrm{~s}$. To test whether TOG5-MT binding is important for maintaining MT polymerization, we created FL Msps-eGFP constructs containing the mutations that abrogated TOG5-MT lattice binding and examined MT polymerization rescue ability in S2 cells depleted of endogenous Msps. All three mutation constructs partially impaired MT polymerization to rates on par with the $\Delta 5$ construct (Fig. $5 \mathrm{~F}$ ). Collectively, these data indicate that TOG5 HR 0 is important for binding the MT lattice, and this activity is necessary for optimal Mspsmediated MT polymerization.

\section{Positionally conserved TOG domains underlie Msps-mediated MT polymerization} TOG5's unique structure and MT lattice-binding activity contrasts with TOGs 1 and 2, which are architecturally similar and preferentially bind free tubulin heterodimers (Al-Bassam et al., 2006; Slep and Vale, 2007; Ayaz et al., 2012, 2014). To determine whether the tubulin-binding properties of TOG domains along the array affect MT polymerization rates, we created two permuted TOG array constructs, replacing TOG1 with TOG5 and vice versa, and tested their ability to rescue EB1tRFP comet velocity in S2 cells (Fig. 6, A and B). Msps 12341 rescued MT polymerization to rates on par with cells expressing the TOG5 Lateral ${ }^{\mathrm{ME}}, \mathrm{RE}, \mathrm{KE}+\mathrm{HR}^{\mathrm{FE}}$ mutant construct that ablates TOG5-MT binding activity. In contrast, Msps 52345 failed to rescue MT polymerization (Fig. 6 B). These data suggest that exchanging the position of structurally distinct TOG domains negatively affects MT polymerization rates. As TOG1 is architecturally honed to bind free $\alpha \beta$-tubulin and TOG5 HR 0 is positioned to engage lateral tubulin heterodimers on the MT lattice (Fig. 3 C), these data support the hypothesis that TOG domains were maintained throughout evolution in a specific order to promote differential TOG-tubulin binding interactions, which is necessary for Msps function. CLASP and Crescerin are also predicted to contain architecturally distinct TOG domains (Fig. 6 C), suggesting that their TOG arrays may also be honed to regulate TOG-tubulin binding, which could underlie their ability to uniquely regulate MT dynamics parameters.

\section{TOG5 is not required to maintain bipolar mitotic spindle length but regulates mitotic index}

The XMAP215 family not only maintains interphase MT polymerization rates but also is vital for the complex process of bipolar mitotic spindle formation (Cullen et al., 1999; Kosco et al., 2001; Cassimeris et al., 2004; Yasuhara and Oe, 2011). We examined whether TOG5 is important for mitotic spindle regulation by creating Msps-eGFP constructs that either removed TOG5 or contained mutations in HR 0-A that we determined compromise MT lattice binding. We compared the TOG5 constructs to FL Msps and $\Delta 34_{2}$, which fully rescued 
A

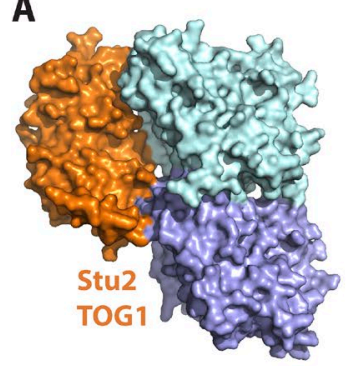

Curved aß-tubulin

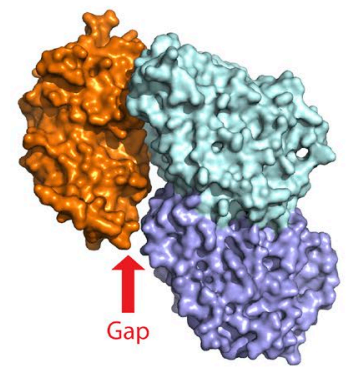

Straight $a \beta$-tubulin

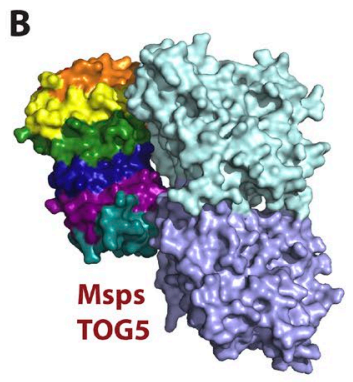

Curved $a \beta$-tubulin

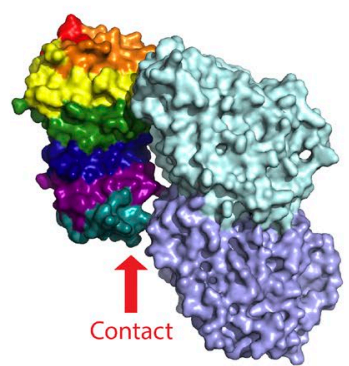

Straight $a \beta$-tubulin

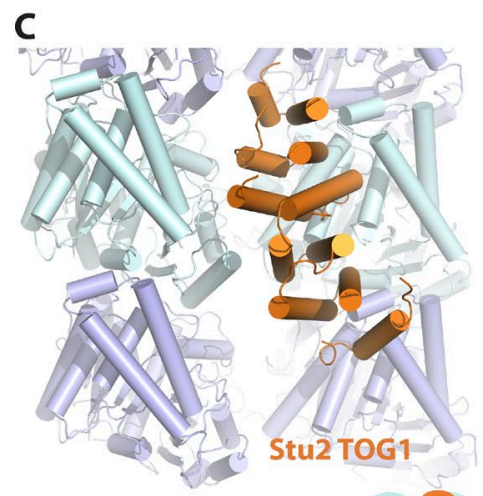

MT-lattice $\alpha \beta$-tubulin No lateral contact

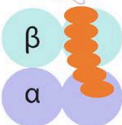

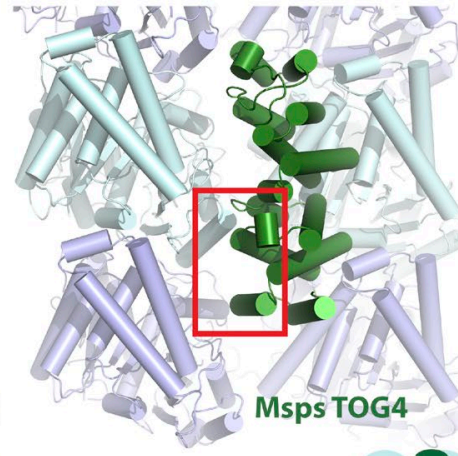

MT-lattice $a ß$-tubulin Lateral contact

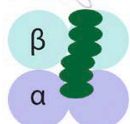

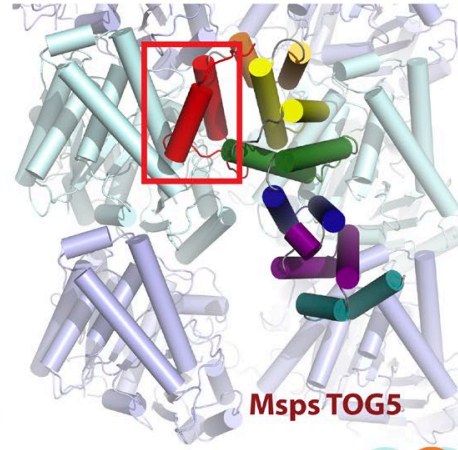

MT-lattice aß-tubulin Lateral contact

Figure 3. TOG5 HR 0 is positioned to engage laterally associated $\boldsymbol{\alpha} \boldsymbol{\beta}$-tubulin subunits on the MT lattice. (A) Stu2 TOG1 bound to a curved (4FFB, left) and straight (modeled, right) $\alpha \beta$-tubulin heterodimer. A gap is formed when TOG1 is superimposed on straight $\alpha \beta$-tubulin (1JFF). (B) Msps TOG5 superimposed on both curved (left) and straight (right) $\alpha \beta$-tubulin using the Stu2 TOG1- $\alpha \beta$-tubulin complex structure as a guide. Although modeling suggests that TOG5 can engage curved $\alpha \beta$-tubulin-like Stu2 TOG1, slight architectural differences in TOG5 uniquely position it to better engage straight $\alpha \beta$-tubulin. (C) TOG1 is not predicted to make lateral $\alpha \beta$-tubulin contacts when superimposed on the MT lattice (left). In contrast, TOG4 HRs D-F (4Y5J, middle) and TOG5 HR $O$ (right) are positioned to contact laterally associated tubulin on the neighboring protofilament (red boxes). Cartoon schematics are depicted at bottom.

MT polymerization velocity (Figs. $7 \mathrm{~A}$ and $1 \mathrm{G}$ ). We first determined whether the reduction in MT polymerization by $\Delta 5$ and HR 0-A mutants (Fig. 5 F) affects mitotic spindle size by measuring the length between bipolar mitotic spindle centrosomes. Control dsRNA-treated S2 cells had a pole-to-pole distance of $7.8 \mu \mathrm{m}$, which contrasted with the short "mini-spindles" (5.7 $\mu \mathrm{m})$ phenotype found in Msps-depleted cells (Fig. 7 B). Expression of FL Msps rescued spindle length in Msps knockdown cells. Mitotic spindle length was also rescued in cells expressing $\Delta 5$ and TOG5 mutant constructs, even though these mutations significantly reduced interphase MT polymerization rates (Fig. 7 B). Interestingly, although $\Delta 34_{2}$ cells rescue MT polymerization (Fig. $1 \mathrm{G}$ ), mitotic spindles in $\Delta 34_{2}$-expressing cells were significantly shorter than spindles in either control or $\Delta 5$ cells, which is consistent with previously reported data examining XMAP215 mutants in Xenopus egg extracts (Reber et al., 2013). We next assessed whether the percentage of mitotic cells, or mitotic index, was affected. msps dsRNA-treated cells had a twofold increase in the percentage of mitotic cells $(6.9 \%)$ compared with control cells $(3.2 \%)$, which could be partially rescued by FL Msps (4.7\%) and $\Delta 34_{2}$ (5.0\%; Fig. 7 C). Surprisingly, $\Delta 5$ had no rescue activity, and instead resulted in a dramatic gain-of-function increase in the mitotic index to $9.4 \%$, surpassing the mitotic index of Msps-depleted cells (Fig. 7 C). Constructs with mutations in TOG5 also failed to rescue the mitotic index, though they did not induce the highly elevated mitotic index observed with the $\Delta 5$ construct (Fig. 7 C). These data indicate that TOG5-MT lattice binding is critical for mitotic function and suggests that cells expressing Msps constructs lacking functional TOG5 either are proceeding through mitosis more slowly or are activating the spindle assembly checkpoint (SAC) because of a failure to form a proper mitotic spindle.

TOG5-MT lattice binding is essential for Msps-mediated mitotic spindle formation To assess whether cells expressing $\Delta 5$ or TOG5 HR 0-A mutant constructs form proper mitotic spindles, we scored mitotic spindle structure using four general phenotypic categories: bipolar/monastral bipolar, misaligned, monopolar, and multipolar (Fig. 8, A and B). Mitotic spindles in control dsRNA-treated cells were $57.5 \%$ bipolar/monastral bipolar and $42.5 \%$ abnormal (misaligned, monopolar, or multipolar). In Msps-depleted cells, the percentage of abnormal spindles increased dramatically to $74.5 \%$ (Fig. 8 B). The fraction of normal bipolar/ monastral bipolar spindles in msps dsRNA-treated cells was rescued by transfection of FL Msps or $\Delta 34_{2}$. Rescue activity was diminished when TOG5 was deleted, yielding aberrant spindle structures in $58.2 \%$ of mitotic cells (Fig. 8 B). Surprisingly, mutations in TOG5 HRs $0-A$ compromised rescue activity even more severely than the $\Delta 5$ construct. When residues implicated in binding laterally associated $\alpha \beta$-tubulin on the MT lattice (Lateral ${ }^{\mathrm{ME}}, \mathrm{RE}, \mathrm{KE}$ ) were mutated, the population of aberrant spindles increased even further, to $69.8 \%$. Relative to $\Delta 5$ expressing cells, the Lateral ${ }^{\mathrm{ME}}, \mathrm{RE}, \mathrm{KE}_{-}$expressing cells showed 


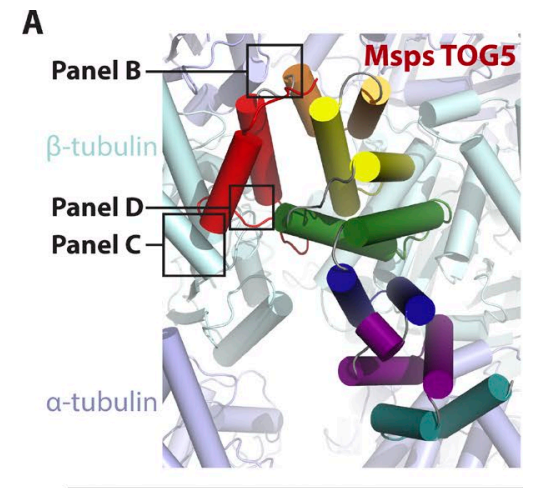

B

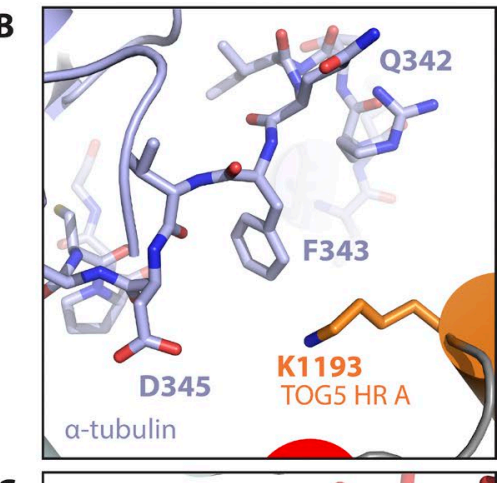

C

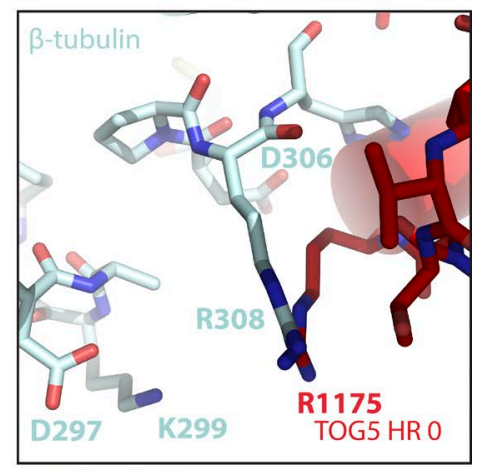

D

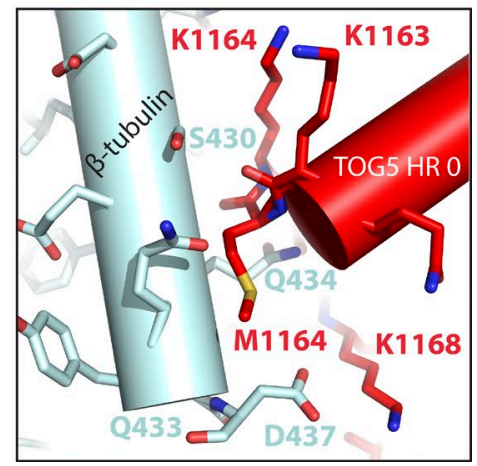

Figure 4. TOG5 K1193, R1175, and M1 164 contact laterally associated $\boldsymbol{\alpha} \boldsymbol{\beta}$-tubulin subunits on the MT lattice. (A) Msps TOG5 superimposed on the MT lattice using the Stu2 TOG1- $\alpha \beta$-tubulin (4FFB) and Mal3-GTP $\gamma$ S MT $(4 A B O)$ complex structures as guides. Boxed regions are shown in zoom view in B-D. (B) TOG5 HR A K1 193 is positioned to interact with the lateral $+1 \alpha$-tubulin subunit. (C and D) TOG5 HR 0 R 1175 (C) and M1164 (D) are positioned to interact with the lateral $\beta$-tubulin subunit.

an increase in the percentage of monopolar spindles (25.9\%), a level similar to that observed in Msps-depleted cells. When the Msps TOG5 HR A residue F1204 was mutated to glutamate $\left(\mathrm{HR} \mathrm{A}^{\mathrm{FE}}\right)$, the population of aberrant spindles was also elevated $(67.4 \%)$, dominated by a dramatic increase in the percentage of misaligned spindles (45.3\%; Fig. 8 B). When TOG5 HR A F1204 was mutated in conjunction with M1164, R1175, and $\mathrm{K} 1193$ (Lateral $^{\mathrm{ME}, \mathrm{RE}, \mathrm{KE}}+\mathrm{HR} \mathrm{A}^{\mathrm{FE}}$ ), the profile of mitotic phenotypes mirrored that of the single F1204E mutant, indicating that TOG5-HR A tubulin binding plays a dominant role in TOG5 function. All Msps constructs localized to centrosomes and spindle MTs at the resolution limit of this experiment, meaning that any phenotypic abnormalities were not caused by localization defects (Fig. 8 A). These data suggest that the binding of TOG5 HRs 0-A to the MT lattice is essential for high-fidelity bipolar mitotic spindle formation, as mutating TOG5-tubulin binding residues yield mitotic spindle phenotype distributions that are more severe than simply deleting TOG5 from the array.

Made depletion rescues the mitotic index of cells expressing $\Delta 5$ or HR O-A mutant constructs

We next determined whether the elevated mitotic index observed with the $\Delta 5$ and mutant TOG5 constructs was a result of $\mathrm{SAC}$ activation. To test this, we codepleted Msps and the SAC component Mad2 in S2 cells, then transfected cells with $\Delta 5$ or mutant TOG5 Msps constructs and scored the mitotic index. Mad2-depleted cells had a mitotic index of $3.7 \%$, which was not significantly different from that of control dsRNA-treated cells (Figs. $7 \mathrm{C}$ and $9 \mathrm{~A}$ ). As a positive control for Mad2 depletion, we assayed mitotic index in KLP61F-depleted cells with and without Mad2 codepletion to ensure that we could effectively silence the SAC. As noted in previous studies, KLP61F depletion dramatically increased the percentage of mitotic cells (9.9\%; Heck et al., 1993; Goshima and Vale, 2005). Codepletion of Mad2 and KLP61F released the SAC, returning the mitotic index to a baseline $3.5 \%$ (Fig. 9 A). When Mad2 was codepleted along with Msps in cells expressing the $\Delta 5$ construct, the percentage of mitotic cells decreased to the control baseline of $3.5 \%$ (Fig. 9 A). Mad2 depletion also suppressed the increase in mitotic index in Msps-depleted cells expressing the TOG5 Lateral $^{\mathrm{ME}, \mathrm{RE}, \mathrm{KE}}+\mathrm{HR} \mathrm{A}^{\mathrm{FE}}$ mutant construct. Collectively, these data suggest that TOG5 is important for regulating mitosis. If TOG5 is removed from the TOG domain array or TOG5-tubulin binding residues are mutated, the SAC is activated as a result of failure to form a proper mitotic spindle, which in turn increases the percentage of mitotic cells.

\section{iscussion}

Recent recognition that XMAP215, CLASP, and Crescerin TOG domains have unique architectures raised questions regarding how TOG domains are used to differentially engage tubulin and affect MT dynamics. We used the Msps pentameric TOG array as a model to investigate the impact of differential TOG-tubulin binding on MT polymerization and mitotic spindle formation. TOG5 was the focus of our efforts. TOG5 was predicted to be similar to ZYG-9 TOG3 by sequence analysis. Our structure of Msps TOG5 confirmed that TOG5 is structurally similar to ZYG-9 TOG3 and, like ZYG-9 TOG3, contains an additional N-terminal HR. This structural finding and sequence analysis suggested that the ZYG-9 trimeric TOG array was akin to a Msps TOG2-TOG2-TOG5-like array. Because prior work demonstrated that TOG1 and TOG2 are functionally equivalent (Ayaz et al., 2014), we tested, and confirmed, that a minimal Msps TOG1-TOG2-TOG5 array could fully 

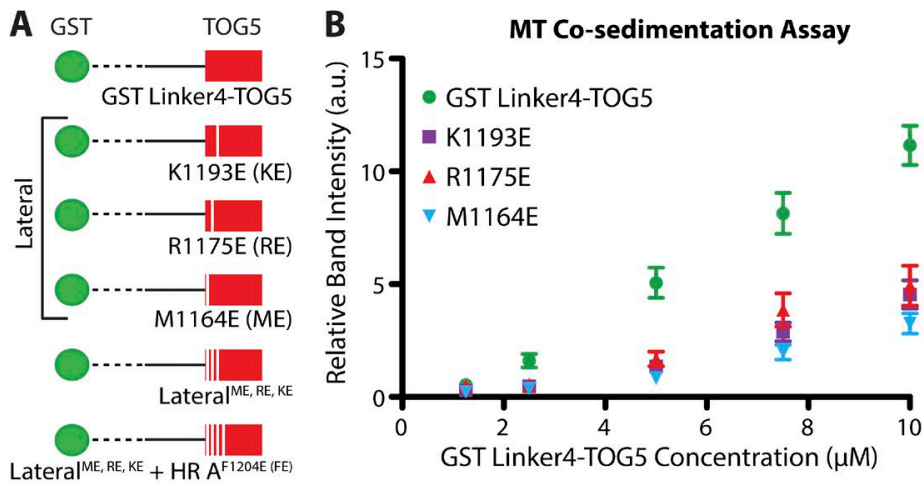

D Cellular MT Localization Assay
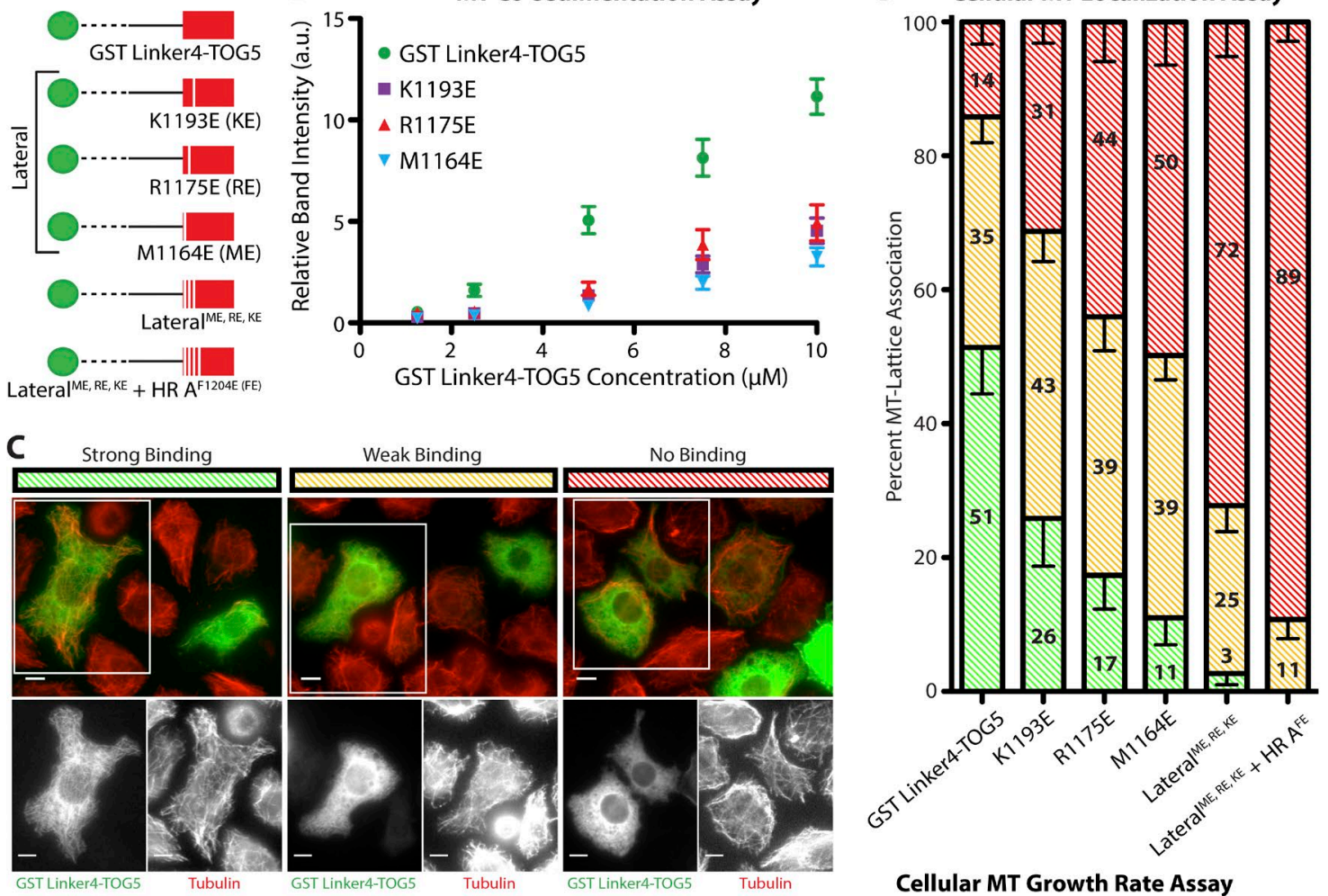

$\mathbf{E}$

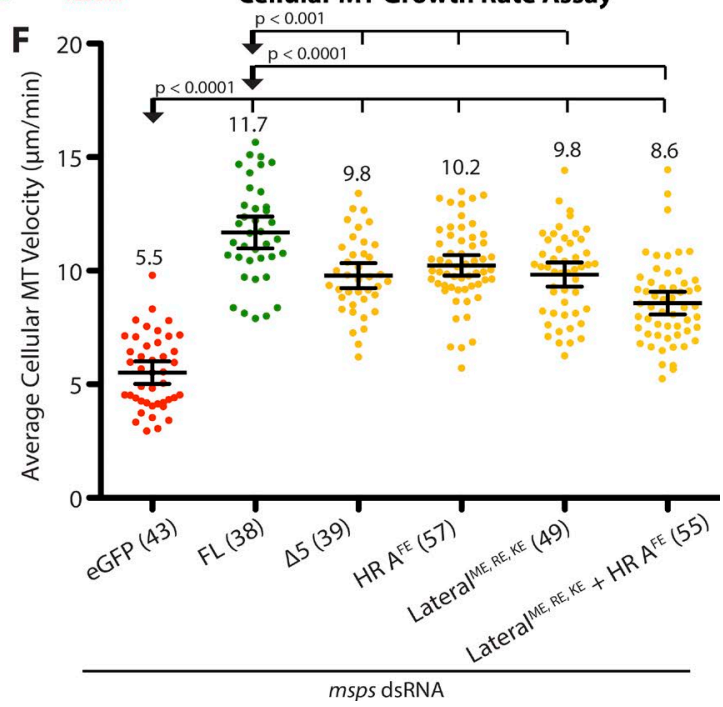

Figure 5. TOG5 uses lateral tubulin-binding determinants to bind the MT lattice, nucleate MTs, and maintain cellular MT polymerization rates. (A) GST dimerized Linker4-TOG5 $(1,085-1,411)$ constructs used for MT co-sedimentation and cellular MT localization assays. (B) GST Linker4-TOG5 co-sediments with taxol-stabilized MTs. Mutating K1 193, R1 175, or M1 164 to glutamic acid reduces the apparent affinity of GST Linker4-TOG5 for the MT lattice. Mean \pm SEM are shown $(n=5)$. (C) Representative immunofluorescence images of strong, weak, or no lattice binding in GST Linker4-TOG5 transfected S2 cells. Bars, 5 rm. (D) GST Linker4-TOG5 robustly associates with MTs in S2 cells. Mutating residues in HR 0, HR A, or HRs 0-A decreases the MT-lattice association activity of GST Linker4-TOG5. Mean \pm SEM are shown $(n=3)$. (E) TOG domains differentially promote MT nucleation and polymerization in a light scattering assay. TOGs 1-3 sequester tubulin, increasing the lag time before MT nucleation and subsequent polymerization. In contrast, TOGs 4 and 5 decrease MT polymerization lag time. Mean \pm SEM are shown $(n=3)$. (F) Mutating TOG5-MT binding determinants prevents full rescue of cellular MT polymerization rates. MT polymerization rates for the three mutants are similar to the rates obtained with the Msps $\Delta 5$ construct. Number of cells analyzed is shown in parentheses. Mean $\pm 95 \%$ confidence intervals are shown; two-tailed Mann-Whitney $U$ test.

drive MT polymerization in cell culture. This highlights the key mechanistic roles these three TOG domains play in XMAP215 family activity and the evolutionary pressure to maintain these domains across species. Because TOGs 3 and 4 were not necessary for Msps-meditated MT polymerization, we tested their role in mitotic regulation. Interestingly, TOGs 3 and 4 are not essential for mitotic spindle assembly and progression through mitosis, but they are required to maintain mitotic spindle length. Why TOGs 3 and 4 regulate spindle length and what roles they play in the XMAP215 mechanism remain to be determined, but they may affect other MT dynamics parameters, such as rescue.

TOG5's unique structure and its conservation with ZYG-9 TOG3 suggested that its distinct element, HR 0, could underlie TOG5's role in XMAP215 function. TOG5 HR 0 is positioned 
A
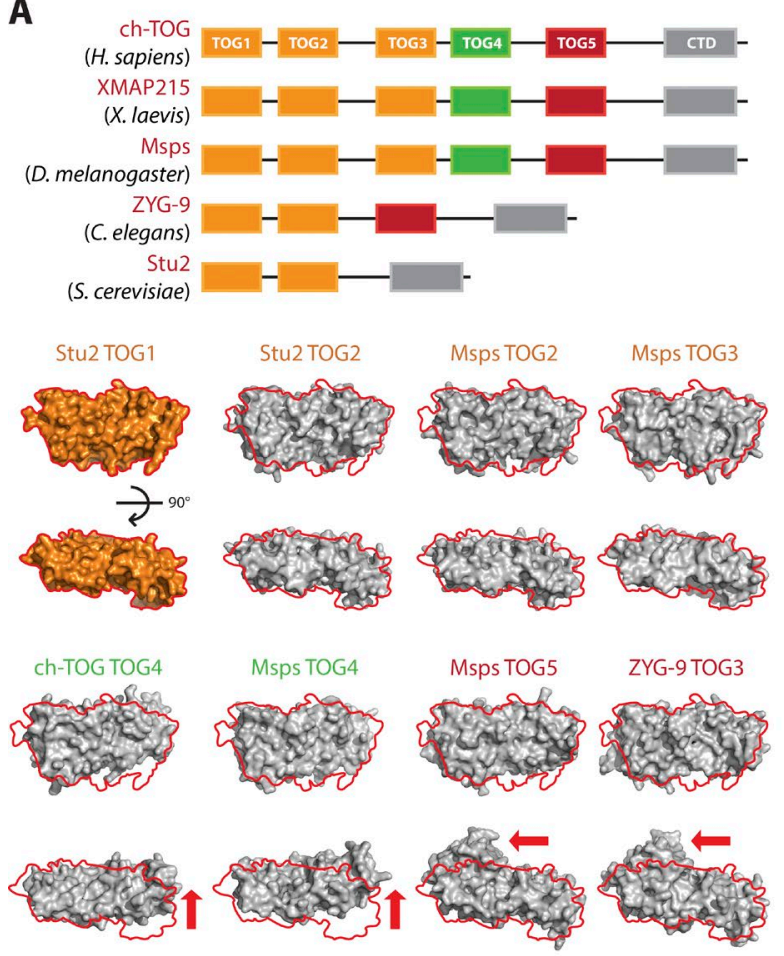

C
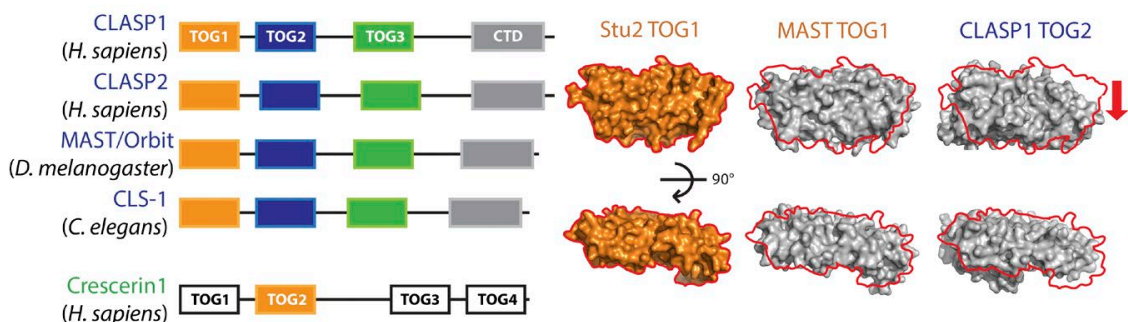

msps dsRNA
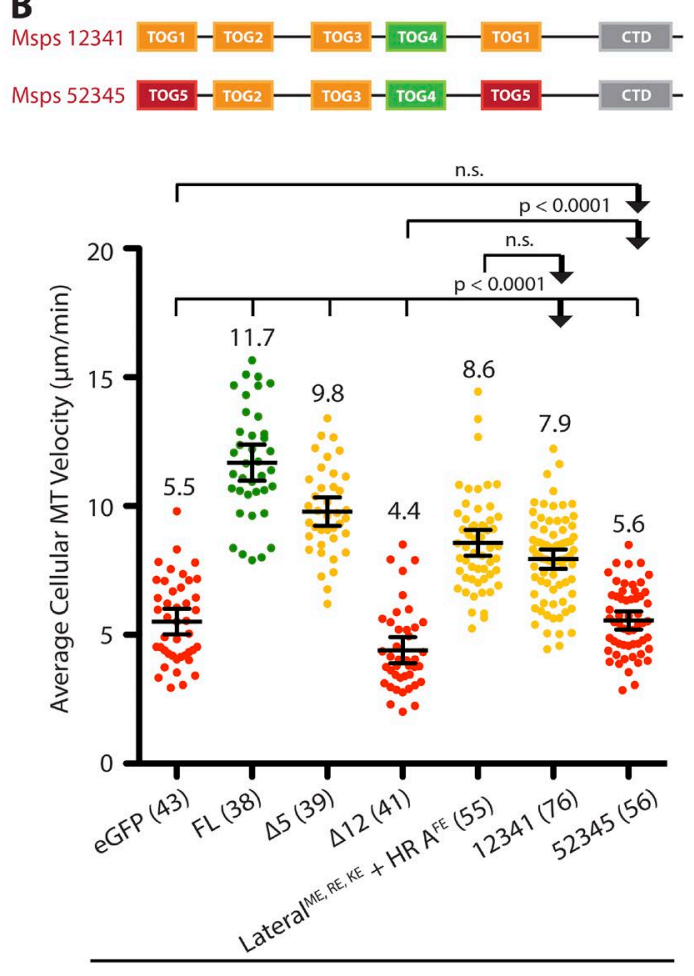

Figure 6. TOG domain order within an array underlies Msps-mediated MT polymerization. (A) Pairwise alignment of Stu2 TOG1 (4FFB) with other XMAP215 TOG domain structures shows that TOG architectures are unique within an array, but are positionally conserved. TOGs 1-3 are structurally similar (orange). In contrast, TOG4 has a large shift across its last three HRs (green, arrow). Interestingly, ZYG-9 TOG3 and TOG5 have HR 0 that extends away from the body of the domain (red, arrow). (B) Shuffling TOG domains differentially affects EB1 comet velocities. Msps 12341 rescued MT polymerization velocity to $7.9 \mathrm{\mu m} / \mathrm{min}$. In contrast, Msps 52345 had no rescue activity. Number of cells analyzed is shown in parentheses. Mean $\pm 95 \%$ confidence intervals are shown; two-tailed Mann-Whitney $U$ test. (C) Although XMAP215 uses a TOG array to promote MT polymerization, other TOG domain-containing MAPs use architecturally distinct arrayed TOGs to differentially regulate MT dynamics. Crescerin promotes MT polymerization in cilia, and CLASP promotes MT pause/rescue in the cytoplasm. We predict that order-specific TOG arrays underlie the ability of these three families to differentially regulate $M T$ dynamics.

to engage lateral $\alpha \beta$-tubulin heterodimers on the MT lattice. We found that TOG5-MT lattice binding was essential for maintaining MT polymerization rates. TOG5 also played a key role in bipolar mitotic spindle formation. When Msps constructs with mutations that affect TOG5-MT binding were expressed in Msps-depleted S2 cells, aberrant mitotic spindles formed, the SAC was triggered, and the mitotic index increased. Previous studies that depleted XMAP215 members found decreased centrosome MT nucleation, defects in MT-kinetochore attachment, and multipolar or malformed spindles (Cullen et al., 1999; Garcia et al., 2001; Popov et al., 2002; Goshima et al., 2005; Cassimeris et al., 2009; Hsu and Toda, 2011). Although these studies focused on depleting XMAP215 family members, our study provides the first evidence that TOG-MT binding affects mitotic spindle formation. The precise mechanistic connection underlying the observations that mutating TOG5 decreased MT polymerization, increased the percentage of aberrant spindle morphologies, and activated the SAC remains unclear. We hypothesize that TOG5 is necessary for MT plus end stabilization, mediated through contacts that engage neighboring protofilaments. These contacts could stabilize the growing MT plus end and limit depolymerization. We also hypothesize that TOG5 may regulate other MAP-MT interactions. Based on our model of TOG5 superpositioned on the MT lattice, we predict that TOG5 can sterically occlude the binding of other MAPs, including EB1 and doublecortin, to the same site on the MT lattice (Fig. S3; Liu et al., 2012; Maurer et al., 2012). This finding corroborates previous studies showing that XMAP215 is positioned plus end from EB1 with a nonoverlapping localization pattern (Nakamura et al., 2012; Maurer et al., 2014). However, TOG5 may potentiate the binding of other MAPs (Ndc-80) or motors (kinesin) to the laterally associated protofilament (Fig. S3). 

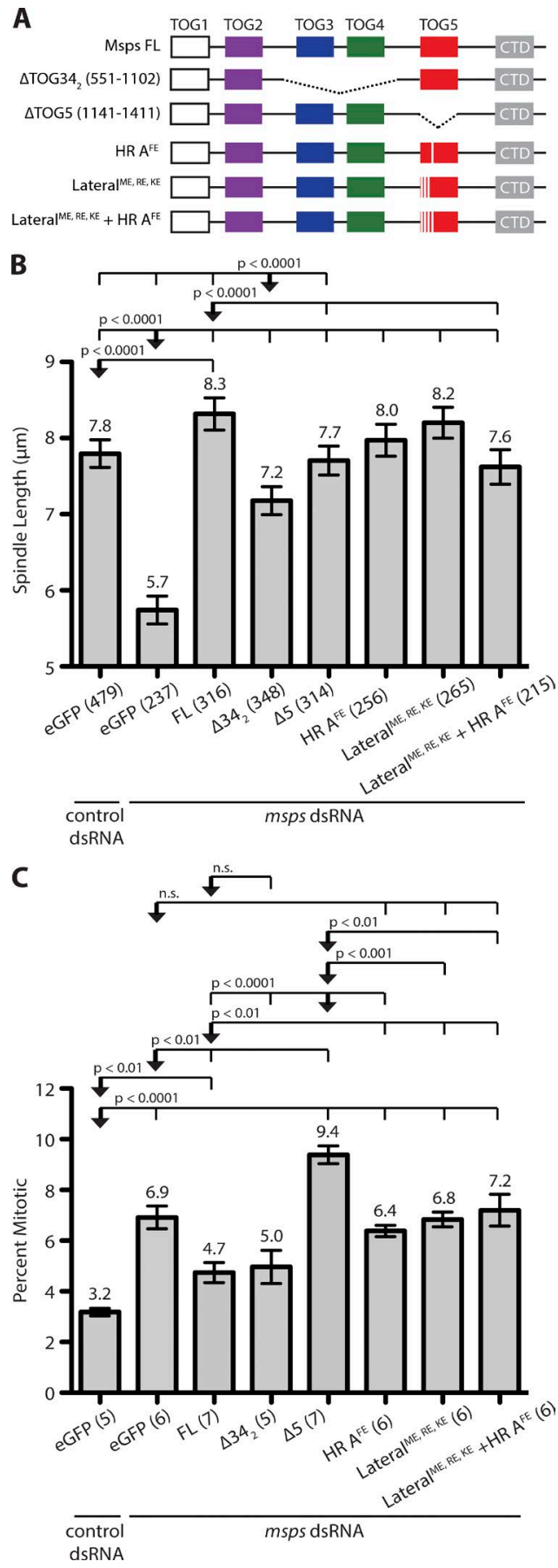

Figure 7. Deleting or mutating TOG5 does not affect mitotic spindle length, but dramatically increases mitotic index. (A) Msps-eGFP constructs analyzed for their ability to rescue mitotic spindle length, mitotic index, and mitotic spindle formation in S2 cells depleted of endogenous Msps. (B) Mitotic spindle pole-to-pole distance in msps dsRNA-treated cells is rescued by transfection of either FL Msps or $\Delta 5$. Mutating residues that compromise TOG5-MT binding do not dramatically affect mitotic spindle pole-to-pole distance. In contrast, $\Delta 34_{2}$ expression only partially rescues pole-to-pole distance. Number of bipolar mitotic spindles measured is shown in parentheses. Mean $\pm 95 \%$ confidence intervals are shown; twotailed Mann-Whitney $U$ test. (C) Deleting TOG5 from the TOG domain array dramatically increases the mitotic index compared with control or $\Delta 34_{2} \mathrm{~S} 2$ cells. Mutating residues involved in TOG5-MT binding increase the mitotic index to levels comparable to endogenous Msps depletion. Number of experiments analyzed is shown in parentheses. Mean \pm SEM are shown; two-tailed unpaired Student's $t$ test.
Previous studies showed that XMAP215 works in concert with the Ndc-80 complex to stabilize/destabilize kinetochores in a tension-dependent fashion and with kinesin to regulate MT dynamics, suggesting there could be synergy between TOG5 and these MAPs/motors (Tournebize et al., 2000; Garcia et al., 2002; Cassimeris et al., 2004; Holmfeldt et al., 2004; Barr and Gergely, 2008; Hsu and Toda, 2011; Rizk et al., 2014; Miller et al., 2016). Probing the synergy between TOG5 and other MAPs/motors is a key next step.

Our structural, modeling, and light scattering data provide evidence that TOG domain architectures predispose TOG domains to bind distinct tubulin states: free, lattice-incorporated, or an intermediary transition state. TOGs 1-3 did not contact lateral tubulin when modeled on the MT lattice, but TOG4 and TOG5 were positioned to engage laterally associated tubulin. Furthermore, incubating TOG 1, 2, or 3 with free tubulin inhibited or increased MT polymerization lag time, an activity that can be ascribed to binding and sequestering free tubulin (Slep and Vale, 2007; Ayaz et al., 2012). On the other hand, TOG4 and TOG5 decreased MT polymerization lag time, suggesting that these domains may stabilize lateral tubulin interactions that promote MT nucleation and limit catastrophe events. The ability of XMAP215 to promote MT nucleation remains controversial (Popov et al., 2002; Reber et al., 2013; Wieczorek et al., 2015). Thus, further examination of the ability of TOG4 and TOG5 to enhance MT nucleation from centrosomes and GMPCPP seeds are important next steps to understanding XMAP215 function.

The differential binding of TOGs $1-5$ to either free or MT lattice-incorporated tubulin supports the model that XMAP215 is polarized and positioned to template MT plus ends (Fig. 9 B). In this model, the CTD localizes XMAP215 to MTs via the EB1 binding protein SLAIN2/Sentin, positioning the CTD distal to the growing plus end, where tubulin is in a nucleotide hydrolysis transition/post-hydrolysis state (Maurer et al., 2011; van der Vaart et al., 2011; Li et al., 2012; Zanic et al., 2013). XMAP215 TOG domains are positioned plus end to the CTD, where TOGs 1 and 2 bind and promote free tubulin incorporation into growing MTs, TOG5 engages MT lattice-incorporated tubulin, and bridging TOG domains (TOG3 and TOG4) stabilize a structural transition state of polymerizing tubulin. The lack of complete rescue activity for the permuted TOG 12341 and 52345 constructs suggests that a polarized positioning of TOG domains is essential for XMAP215 function. The TOG 12341 construct rescued MT polymerization to rates on par with a FL Msps construct where TOG5 MT binding activity was ablated. Furthermore, positioning a MT-lattice binding TOG domain (TOG5) in place of TOG1 did not support MT polymerization, indicating that TOG5 MT-binding activity plays a unique, position-specific role in the array. These data together with our TOG domain deletion experiments suggest that XMAP215 does not wrap around and add a single tubulin heterodimer processively to MT plus ends (Al-Bassam et al., 2006; Brouhard et al., 2008). Instead, XMAP215 is positioned to add multiple tubulin heterodimers to the plus end in a templating fashion (Spittle et al., 2000; Kerssemakers et al., 2006; Slep and Vale, 2007). Whether XMAP215 family members use their TOG domains to cooperatively add tubulin to MT plus ends or use a tethered delivery mechanism remains to be determined (Ayaz et al., 2014).

Our findings support a TOG array paradigm in which TOG domains are used to bind to, or promote, structurally distinct states of tubulin subunits along a reaction coordi- 
A

control dsRNA

msps dsRNA

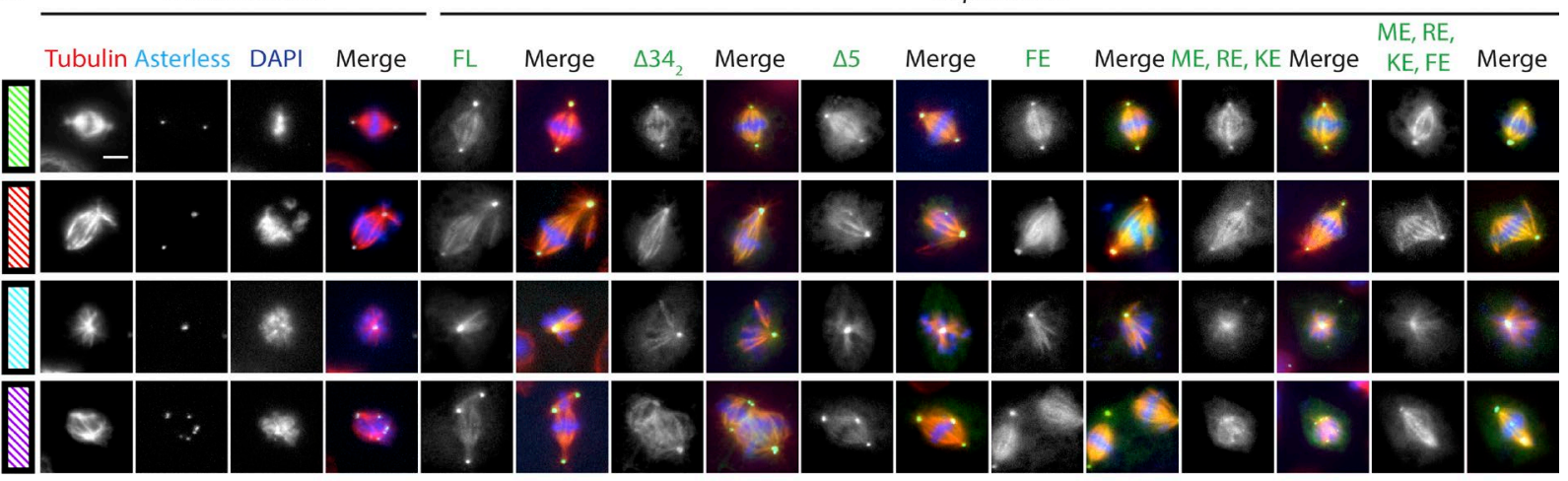

B

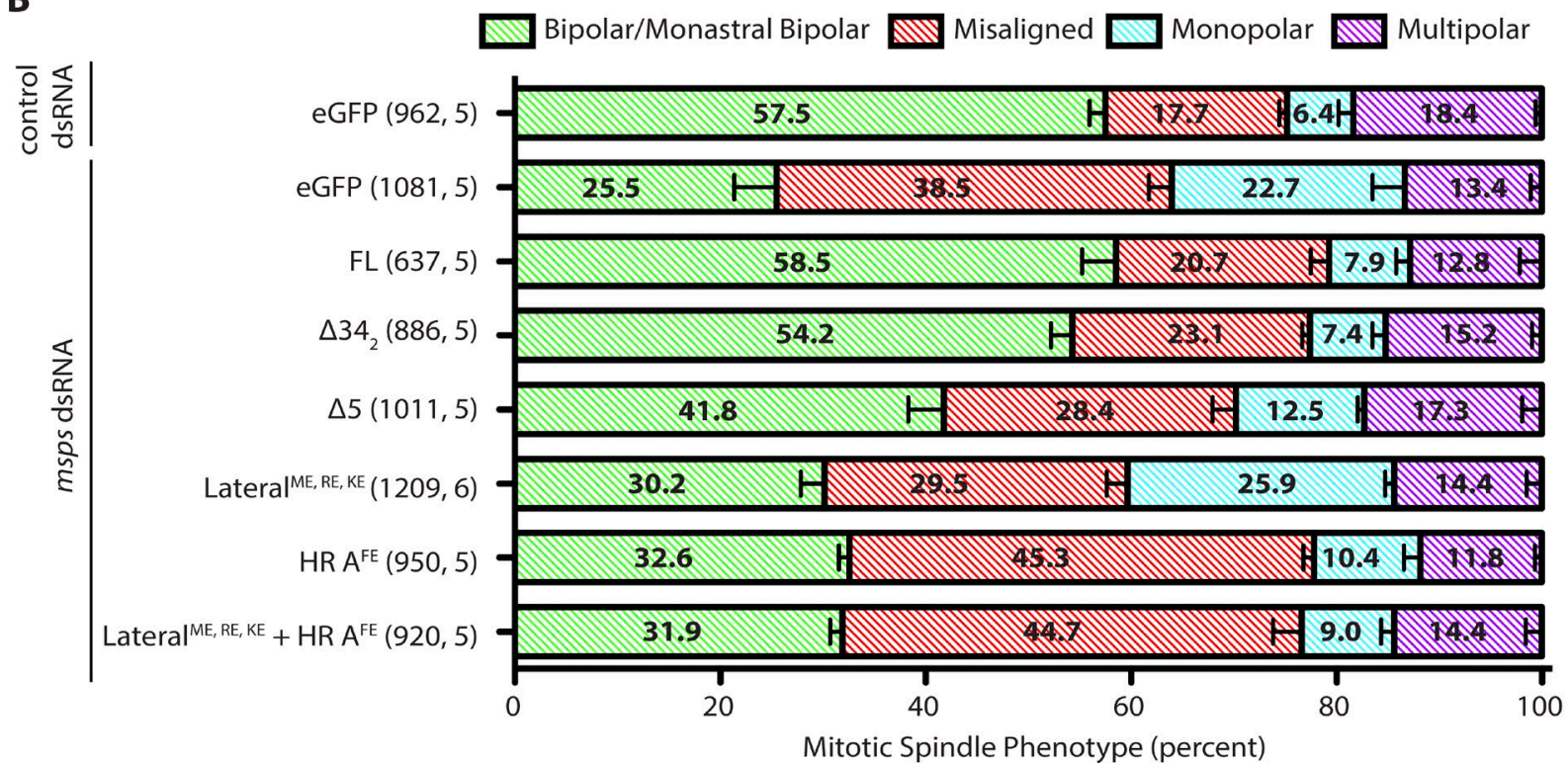

Figure 8. TOG5 is essential for proper mitotic spindle formation. (A) Representative immunofluorescence images of the four mitotic phenotypes categorized in S2 cell mitotic spindle rescue experiments: bipolar/monastral bipolar, misaligned, monopolar, and multipolar. Bar, 5 um. (B) Quantification of mitotic phenotypes for Msps depletion and rescue experiments. Depleting Msps decreases the number of bipolar/monastral bipolar mitotic spindles compared with control cells, with a concomitant increase in aberrant spindle phenotypes including misaligned and monopolar. Transfection of FL Msps or $\Delta 34_{2}$ rescues mitotic spindle defects. Deleting TOG5 or mutating TOG5-MT binding residues results in a dramatic decrease in the ability of these constructs to rescue bipolar/monastral bipolar mitotic spindle formation. The percentage of misaligned and monopolar mitotic spindles increases for mutation constructs containing F1204E (HR A ${ }^{\mathrm{FE}}$ ) and lateral tubulin-binding mutations (LateralME, ${ }^{\mathrm{RE}}{ }^{\mathrm{KE}}$ ), respectively. Number of mitotic cells analyzed and number of independent experiments are shown in parentheses. Mean \pm SEM are shown.

nate to regulate MT dynamics. Although the XMAP215 family uses four to five TOG domains to drive MT polymerization, other TOG domain-containing MAPs regulate additional MT dynamic states. The CLASP family promotes MT pause/rescue using a trimeric TOG array (Akhmanova et al., 2001; Bratman and Chang, 2007; Al-Bassam et al., 2010; De la Mora-Rey et al., 2013; Leano et al., 2013; Maki et al., 2015). A new family of TOG domain-containing proteins, termed Crescerin, regulates MT polymerization in a set of primary cilia (Das et al., 2015). XMAP215 TOGs 1-3, MAST TOG1, and Crescerin TOG2 have similar structures, suggesting that these domains may preferentially bind tubulin's curved state found in solution (Ayaz et al., 2012, 2014; De la Mora-Rey et al., 2013; Das et al., 2015; Howard et al., 2015). In contrast, XMAP215 TOG4 has a unique bend across its last three HRs (Fox et al., 2014), which we hypothesize positions TOG4 to contact lateral tubulin in a neighboring protofilament. TOG4's architecture is similar to that of CLASP2 TOG3, suggesting that these domains may bind a similar form of polymerized tubulin (Maki et al., 2015). As described, XMAP215 TOG5 has a distinct architecture that engages laterally associated tubulin subunits on the MT and bridges protofilaments. It is interesting to note that potential lateral binding conferred by TOG4 cannot fully substitute for TOG5 lateral binding activity, suggesting that TOG4 and TOG5 may be honed to affect different phases of MT dynamics. CLASP1 TOG2 has a unique bent architecture predicted to engage tubulin in a hypercurved state that may promote MT pause (Leano et al., 2013). If architecture underlies TOG-tubulin binding specificity, we predict that permuting TOG domain order across arrays could be used to differentially affect MT dynamics. Furthermore, the localization of TOG-containing proteins to distinct cellular regions such as the cytoplasm or cilia suggests that these families may be designed to optimally modulate MT dynamics in unique cellular spaces where specific tubulin isoforms or tubulin 
A

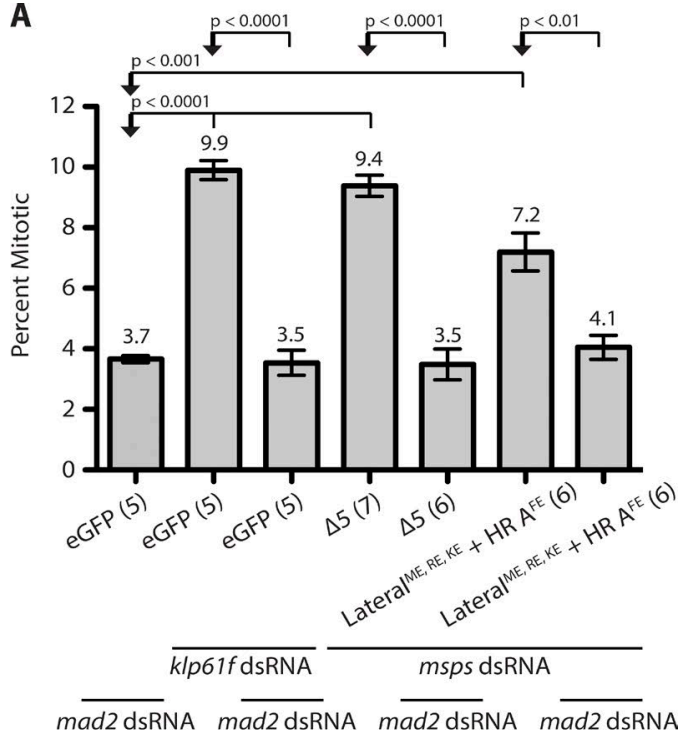

B

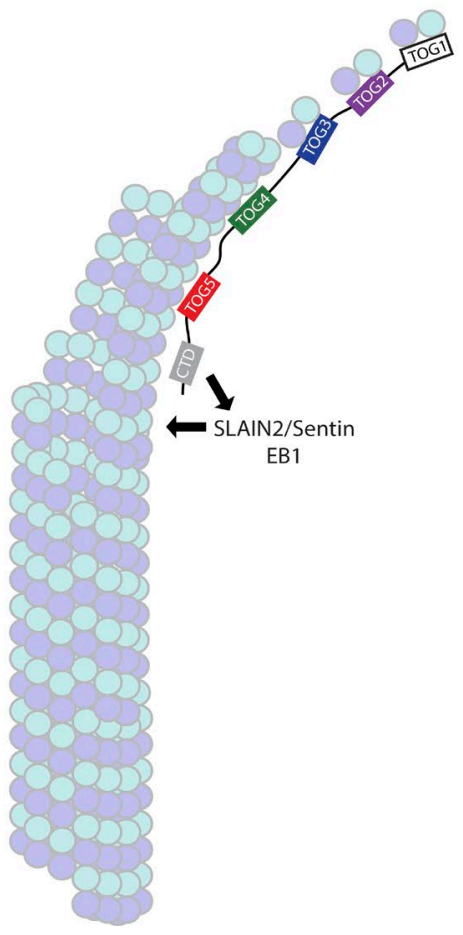

Figure 9. Deleting or mutating TOG5 triggers the SAC. (A) Although Msps $\Delta 5$ or Lateral ${ }^{\mathrm{ME}}, \mathrm{RE}, \mathrm{KE}+\mathrm{HR} \mathrm{A}^{\mathrm{FE}}$ mutant constructs increase the mitotic index in S2 cells depleted of endogenous Msps, codepletion of the SAC protein Mad2 restores the mitotic index to control levels (3.2\%, Fig. $6 \mathrm{C}$ ). Mad2 depletion also rescues the elevated mitotic index observed when KLP61F (a canonical SAC activator) is depleted. Number of independent experiments is shown in parentheses. Mean \pm SEM are shown; two-tailed unpaired Student's $t$ test. (B) Model of Msps at MT plus ends. Msps TOGs $1-3$ are modeled interacting with free $\alpha \beta$-tubulin subunits, promoting their incorporation into the growing MT plus end. Msps TOGs 4-5 engage lattice-incorporated tubulin in the polymer, where they enhance protofilament-protofilament stabilization. The Msps CTD binds the sentin-EB 1 complex, which positions the CTD at the MT lattice region undergoing GTP hydrolysis (MT structure adapted from Guesdon et al., 2016).

posttranslational modifications dominate. Generating novel synthetic TOG arrays and redirecting MAP localization to regions of the cytoplasm, the spindle, or the cilium are new areas of study that pose questions yet to be explored.

\section{Materials and methods}

\section{Multiple species alignment}

Species used to determine TOG5 conservation included human (chTOG, Homo sapiens), chimpanzee (CKAP5, Pan troglodytes), cow (CKAP5, Bos taurus), dog (CKAP5, Canis familiaris), chicken (CKAP5, Gallus gallus), mouse (CKAP5, Mus musculus), frog (XMAP215, Xenopus laevis), fly (Msps, Drosophila), thale cress (MOR1, Arabidopsis thaliana), tobacco (TMBP200, Nicotiana tabacum), and slime mold (CP224, Dictyostelium discoideum). CLU STALW produced preliminary alignments that were adjusted manually (Thompson et al., 1994). Ce ZYG-9 TOG3 was added to the alignment to determine conservation across TOG5 domains and ZYG-9 TOG3.

\section{Construct cloning and expression}

Drosophila Msps TOG5 (1,141-1,411) and Linker4-TOG5 (1,0851,411) were subcloned into pET28 (EMD Millipore) and pGEX-6P-2 (GE Healthcare) expression vectors containing a $\mathrm{N}$-terminal $\mathrm{His}_{6}$ or GST tag, respectively. TOG5 R1157E, E1161R, M1164E, W1169E,

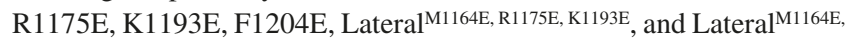
R1175E, K1193E + HR A ${ }^{\mathrm{F} 1204 \mathrm{E}}$ were generated using either a single or multiple rounds of QuikChange mutagenesis (Agilent Technologies). pET28- and pGEX-6P-2-TOG5 constructs were transformed into BL21 DE3 Escherichia coli (pLysS) for expression. Under either kanamycin (pET28) or ampicillin (pGEX-6P-2) selection, 6-liter cultures were grown in luria broth at $37^{\circ} \mathrm{C}$. At a $600-\mathrm{nm}$ optical density of 0.6 , protein expression was induced with $0.125 \mathrm{mM}$ IPTG, and cultures were grown for $20 \mathrm{~h}$ at $18^{\circ} \mathrm{C}$. Cells were harvested by centrifugation at $2,100 \mathrm{~g}$ for $8 \mathrm{~min}$ at $4^{\circ} \mathrm{C}$. Pellets were resuspended in $100 \mathrm{ml}$ buffer A (25 mM Tris, pH 8.0, $300 \mathrm{mM} \mathrm{NaCl}, 10 \mathrm{mM}$ imidazole, and $0.1 \%$ $\beta$-mercaptoethanol) or buffer $\mathrm{G}$ (25 mM Tris, $\mathrm{pH} 8.0,300 \mathrm{mM} \mathrm{NaCl}$, and $0.1 \% \beta$-mercaptoethanol) for pET28 and pGEX-6P-2 constructs, respectively, and stored at $-20^{\circ} \mathrm{C}$.

\section{Protein purification}

Msps TOG5 $(1,141-1,411)$ constructs were purified by $\mathrm{Ni}^{2+}$-NTA affinity chromatography. Bacterial pellets were thawed, and PMSF was added to a final concentration of $1 \mathrm{mM}$. Cells were lysed by sonication and cleared by centrifugation at $23,000 \mathrm{~g}$ for $50 \mathrm{~min}$ at $4^{\circ} \mathrm{C}$. The supernatant was loaded onto a $\mathrm{Ni}^{2+}$-NTA column (QIAGEN) and washed with buffer A. A 250-ml linear gradient of buffer A to buffer B (buffer A with $300 \mathrm{mM}$ imidazole) eluted the protein. Fractions deemed pure by SDS-PAGE were pooled, and the $\mathrm{His}_{6}$ tag was removed by PreScission Protease for $16 \mathrm{~h}$ at $4^{\circ} \mathrm{C}$ during dialysis into buffer A. A subsequent $\mathrm{Ni}^{2+}$-NTA column at $4{ }^{\circ} \mathrm{C}$ removed PreScission Protease and uncleaved $\mathrm{His}_{6}$ tagged protein. Cleaved protein was dialyzed into storage buffer (10 mM Hepes, pH 7.0, $150 \mathrm{mM} \mathrm{NaCl}$, and $0.1 \% \beta$-mercaptoethanol), concentrated to $15 \mathrm{mg} / \mathrm{ml}$, flash-frozen in liquid nitrogen, and stored at $-80^{\circ} \mathrm{C}$. The $\mathrm{His}_{6}$ tag on native and mutant TOG5 $(1,141-1,411)$ protein used for $\mathrm{CD}$ was not cleaved. Instead, pure fractions were dialyzed into storage buffer and stored as described earlier.

Purification of GST tagged Linker4-TOG5 $(1,085-1,411)$ constructs followed the described protocol with the following alterations. The supernatant was loaded onto a glutathione-Sepharose column (GE Healthcare), washed with buffer $\mathrm{G}$, and batch eluted with buffer HG (buffer $\mathrm{G}$ containing $25 \mathrm{mM}$ glutathione). Pure protein was dialyzed into storage buffer and stored according to the described protocol.

\section{Crystallization}

Msps TOG5 $(1,141-1,411)$ crystallization followed the hanging drop vapor diffusion method. Drops included $2 \mu \mathrm{l}$ protein at $15 \mathrm{mg} / \mathrm{ml}$ and $2 \mu \mathrm{l}$ of a $500-\mu \mathrm{l}$ well solution (26\% PEG 3350 and $0.15 \mathrm{M}$ so- 
dium tartrate dibasic dihydrate). After $1 \mathrm{~d}$ of equilibration at $20^{\circ} \mathrm{C}$, small rod-shaped TOG5 crystals emerged. Crystal quality was enhanced using both streak- and microseeding before drop equilibration. TOG5 crystals were transferred into a cryoprotection solution containing the mother liquor supplemented with $30 \%$ glycerol and flash-frozen in liquid nitrogen.

\section{Data collection, structure determination, and refinement}

Isomorphous Msps TOG5 single crystal diffraction data were collected at the Advanced Photon Source (Argonne National Laboratory, Lemont, IL) GM/CA-CAT 23-IDB beamline. The dataset was collected at a wavelength of $1.03319 \AA$ with an exposure time of $1 \mathrm{~s}$ and $0.35^{\circ}$ oscillations for a total of 283 frames. The data were indexed, integrated, and scaled using XDS to a resolution of $2.00 \AA$. Msps TOG5 was solved by the AutoMR molecular replacement method (Phenix) using C. elegans ZYG-9 TOG3 (Protein Data Bank accession no. 2OF3) as a search model (Adams et al., 2010; Kabsch, 2010). The resulting model was refined using iterative manual building in Coot and refinement with phenix.refine (Adams et al., 2010; Emsley et al., 2010). Structure refinement was monitored using free R, with $4.5 \%$ of the data randomly excluded from refinement. APBS and the Dali pairwise alignment server were used to calculate electrostatics, pairwise alignments, and root-mean-square deviation values (Baker et al., 2001; Hasegawa and Holm, 2009).

\section{Cell culture and dsRNA generation}

Drosophila S2 cells were cultured and treated with dsRNA as described previously (Rogers and Rogers, 2008). In brief, S2 cells were cultured in Sf900 II SFM medium supplemented with 1× Anti-Anti (Gibco) and split every 3-5 d. DNA templates for dsRNA synthesis were amplified from pBlueScript (Addgene) vector (control) or S2 genomic DNA (msps 5' and 3' UTR, mad2, and klp61f). Primers also included a nonspecific DNA sequence, a SalI (forward primer) or KpnI (reverse primer) restriction site, and the T7 RNA polymerase recognition site. Primers are as follows: control dsRNA: forward, 5'-GACTATCGTGTCGACTAATACGACTCACTATAGGAAATT GTAAGCGTTAATATTTTG-3', and reverse, 5'-GACTATCGTGGT ACCTAATACGACTCACTATAGGAACAGTTGCGCAGCCTGAAT GG-3'; msps 5'-UTR: forward, 5'-GACTATCGTGTCGACTAATAC GACTCACTATAGGTGTGAGTAGCGGTCACACTG-3' and reverse, 5'-GACTATCGTGGTACCTAATACGACTCACTATAGGCA TCAGAATTGTGATCCAAGTACC-3'; msps 3'-UTR: forward, 5'-GA CTATCGTGTCGACTAATACGACTCACTATAGGACTGTGCG CTTCCCGTAGCTA-3', and reverse, 5'-GACTATCGTGGTACC TAATACGACTCACTATAGGCGCATATAGTTCATGAGGATG-3'; mad2: forward, 5'-GACTATCGTGTCGACTAATACGACTCACTA TAGGAATGGCTCTCGAAGAACATGATC-3', and reverse, 5'-GAC TATCGTGGTACCTAATACGACTCACTATAGGTTAAGTGCTCA TCTTGTAGTTGACC-3'; and klp61f: forward, 5'-GACTATCGT GTCGACTAATACGACTCACTATAGGGACTCTGACATTGGCAT CATACC-3', and reverse, 5'-GACTATCGTGGTACCTAATACGAC TCACTATAGGTTCGGTGTATTCCTTGAGC-3'.

For ease of dsRNA DNA template generation, PCR products were subcloned into pUC19 vector (New England Biolabs, Inc.) and sequenced. PCR product $(10 \mu \mathrm{g})$ generated using pUC19 plasmid and the aforementioned primers was incubated with reaction buffer (400 mM Hepes-KOH, pH 7.5, $120 \mathrm{mM} \mathrm{MgCl} 2,200 \mathrm{mM}$ DTT, and $10 \mathrm{mM}$ spermidine), rNTPs (Promega), pyrophosphatase (Thermo Fisher Scientific), RiboLock (Thermo Fisher Scientific), and purified T7 RNA polymerase at $37^{\circ} \mathrm{C}$ for $3-5$ d to produce dsRNA. dsRNA reactions were incubated with RQ1 RNase free DNase (Promega) for $1 \mathrm{~h}$ at $37^{\circ} \mathrm{C}$. Resulting dsRNA was stored at $-20^{\circ} \mathrm{C}$.

\section{Drosophila S2 cell expression constructs and transfection}

Expression constructs for full-length EB1:EB1-tRFP and Msps-GFP were described previously (Rogers et al., 2002; Currie et al., 2011; Grode and Rogers, 2015; Trogden and Rogers, 2015). Msps $\Delta$ TOG5 $(1,141-1,411), \quad \Delta$ TOG $34_{1} \quad(551-1,127), \quad \Delta$ TOG34 $_{2} \quad(551-1,102)$, $\Delta$ TOG12 (1-505), $\Delta$ TOG45 (848-1,411), $\Delta$ TOG345 (582-1,411), and $\Delta$ TOG12345 $(1-1,411)$ were generated using primers (Table S1) flanking the removed residues (in parentheses) and KOD polymerase (EMD Millipore). Msps M1164E, R1175E, K1193E, F1204E,

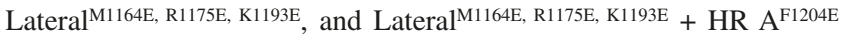
were generated with either a single or multiple rounds of QuikChange mutagenesis (Agilent Technologies). Shuffled Msps TOG 12341 (TOG1: 6-234) and 52345 (TOG5: 1,141-1,411) constructs were generated using Gibson cloning techniques (NEBuilder HiFi DNA Assembly; New England Biolabs, Inc.). Cells were treated with dsRNA for $5 \mathrm{~d}$. On day 3, cells were transfected using FuGENE HD (Promega) according to the manufacturer's instructions. Construct expression was induced $24 \mathrm{~h}$ after transfection with $170 \mu \mathrm{M}$ copper sulfate and imaged or fixed after 18-20 h. Because of the increased transfection difficulty of Mad2 and Msps double-knockdown cells, cells were transfected on the first day of knockdown. Construct induction and cell fixation was completed on days 4 and 5, respectively, as with other single knockdown cells.

\section{Total internal reflection fluorescence microscopy}

Cells were seeded onto $0.5 \mathrm{mg} / \mathrm{ml}$ concanavalin A (ConA)-treated coverslips attached by UV-curable adhesive (Norland Products) to drilled 35-mm tissue culture dishes 45 min before imaging in Schneider's medium supplemented with 10\% FBS (Gibco) and 1× Anti-Anti. Timelapse images were collected with a Nikon Apo TIRF 100× oil/1.49-NA objective lens at RT using a motorized total internal reflection fluorescence system (Nikon) mounted on an inverted microscope (TI; Nikon), controlled by NIS Elements software (Nikon). For EB1 tracking, images were acquired every $3 \mathrm{~s}$ for 4 min with an Andor-Clara Interline camera (Andor Technologies).

\section{EB 1 comet tracking and corrected mean cell fluorescence/pixel calculation}

EB1 comets were tracked manually using the plugin MTrackJ in Fiji (Meijering et al., 2012). To calculate EB1 comet velocities, instantaneous velocities were averaged across the lifetime of an individual EB1 comet ( $30 \mathrm{~s}$ or more). The mean of 10 or more individual EB1 comet velocities were taken per cell. Resulting cell means are displayed using a vertical scatter plot, mean, and $95 \%$ confidence intervals and analyzed using a two-tailed nonparametric Mann-Whitney $U$ test (Prism; GraphPad Software). Cells from three or more independent transfections for each Msps-eGFP construct were tracked for EB1 comet velocity. The corrected mean cell fluorescence/pixel of Msps-eGFP-expressing cells used for EB1-tRFP tracking experiments was obtained by manually outlining cells in Fiji to determine the mean gray value, which was background subtracted using the mean gray value of an area outside of the cell. The resulting corrected mean cell fluorescence/pixel was plotted against EB1 comet velocity in Prism and analyzed using linear regression (Prism). Analyses were completed for 10 or more cells tracked on three or more independent experimental days.

\section{Microtubule co-sedimentation}

MT co-sedimentation experiments were performed with taxolstabilized MTs. In brief, tubulin $(20 \mu \mathrm{M}$ final concentration) was diluted in BRB80 (80 mM Pipes, pH 6.8, $1 \mathrm{mM} \mathrm{MgCl}_{2}$, and $1 \mathrm{mM}$ EGTA) supplemented with $1 \mathrm{mM}$ GTP and $1 \mathrm{mM}$ DTT and allowed to sit on ice for $5 \mathrm{~min}$. The solution was warmed to $37^{\circ} \mathrm{C}$, and taxol was introduced stepwise to generate long stable MT polymers. 1:100 
dilutions of $20 \mu \mathrm{M}, 200 \mu \mathrm{M}$, and $2 \mathrm{mM}$ taxol were added with incubation times of 5, 5, and $15 \mathrm{~min}$, respectively. Msps GST dimerized Linker4-TOG5 $(1,085-1,411)$ constructs were cleared at 50,000 $g$ at $4^{\circ} \mathrm{C}$ for $7 \mathrm{~min}$ and subsequently diluted to $50 \mu \mathrm{M}$ in BRB80. Constructs were diluted to final concentrations of $1.25,2.5,5,7.5$, and $10 \mu \mathrm{M}$ in BRB80, $10 \mu \mathrm{M}$ taxol-stabilized MTs, $1 \mathrm{mM}$ GTP, and $20 \mu \mathrm{M}$ taxol and allowed to incubate for $20 \mathrm{~min}$ at RT. Reactions were layered on top of a glycerol cushion (BRB80, 40\% glycerol, $1 \mathrm{mM}$ GTP, and $20 \mu \mathrm{M}$ taxol) and centrifuged at $70,000 \mathrm{~g}$ for $30 \mathrm{~min}$ at $25^{\circ} \mathrm{C}$. The pellets were resuspended in equal volumes of BRB80, and SDS-PAGE was used to visualize the protein. The resulting gels were scanned, and densitometry was performed to determine the relative intensity of Linker4-TOG5 construct in each pellet. To account for uneven destaining within and across gels, Linker4-TOG5 protein intensity was normalized to a relative tubulin intensity as the concentration of MTs was kept constant. All experiments were completed five times on two or more independent days. Relative band intensities for the amount of pelleted GST Linker4TOG5 construct were plotted with the mean \pm SEM (Prism).

\section{Tubulin polymerization light scattering assay}

Individual TOG domains including TOG1 (1-241), TOG2 (267-505), TOG3 (582-825), TOG4 (848-1,084), and TOG5 $(1,141-1,411)$ were expressed and purified as described earlier, including $\mathrm{His}_{6}$ tag removal. Tubulin polymerization was monitored using a SPEX Fluorolog-3 spectrofluorometer (Horiba Jobin Yvon) in high-voltage (950 V) Tformat mode using excitation and detection wavelengths of $350 \mathrm{~nm}$. Excitation and emission slits were set at 0.5 and $0.75 \mathrm{~nm}$, respectively. Clarified tubulin samples $(18 \mu \mathrm{M}$ final concentration) were incubated for $10 \mathrm{~min}$ at $4{ }^{\circ} \mathrm{C}$ alone or with individual TOG domains $(2 \mu \mathrm{M}$ final concentration) in MES/glycerol polymerization buffer to enhance proper MT structure formation and MT polymerization (50 mM MES, pH 6.6, $3.4 \mathrm{M}$ glycerol, $5 \mathrm{mM}$ DTT, $1 \mathrm{mM}$ EGTA, $5 \mathrm{mM} \mathrm{MgSO}_{4}$, and $1 \mathrm{mM}$ GTP). The cuvette holder was maintained at $37^{\circ} \mathrm{C}$, and samples were injected into a 4-mm-pathlength cuvette. Scattering was recorded for 1,500 s at 1-s intervals. Spikes in scattering during the first $100 \mathrm{~s}$ were caused by sample equilibration. Experiments were completed in triplicate, and mean traces were plotted with SEM error bars (Prism).

\section{Comparative superposition modeling}

The Stu2 TOG1- $\alpha \beta$-tubulin structure (4FFB) was used as a guide to model TOG domain interactions with curved (4FFB), straight (1JFF), and MT lattice-incorporated tubulin (4ABO). In brief, we used $\beta$-tubulin as a guide to align the Stu 2 TOG1- $\alpha \beta$-tubulin structure to the lattice coordinates of straight tubulin (Löwe et al., 2001) and the Mal3-GTP $\gamma \mathrm{S}$ MT EM structure (Maurer et al., 2012). Other TOG domains were then aligned to Stu2 TOG1 in these composite models. TOG domains 2, 3, and 5 were aligned with Stu2 TOG1 across HRs A-F. Because of the unique structure of TOG4 across HRs D-F, TOG4 was aligned with Stu2 TOG1 across HRs A-C.

\section{Circular dichroism}

In a 1-mm-pathlength cuvette, native, R1157E, E1161R, M1164E, W1169E, R1175E, K1193E, F1204E, Lateral ${ }^{\text {M1164E, R1175E, K1193E, or }}$ Lateral $^{\mathrm{M} 1164 \mathrm{E}, \mathrm{R} 1175 \mathrm{E}, \mathrm{K} 1193 \mathrm{E}}+$ HR A ${ }^{\mathrm{F} 1204 \mathrm{E}}$ TOG5 $(1,141-1,411)$ constructs (His ${ }_{6}$ tag present) were diluted to $0.10 \mathrm{mg} / \mathrm{ml}$ in CD buffer (10 $\mathrm{mM}$ sodium phosphate, $\mathrm{pH} 7.4$, and $50 \mathrm{mM}$ sodium fluoride). Spectra were acquired at $20^{\circ} \mathrm{C}$ from $260-185 \mathrm{~nm}$ with a step size of $0.5 \mathrm{~nm}$ every $1.25 \mathrm{~s}$ using a Chirascan-plus CD spectrometer (Applied Photophysics). Subsequently, thermal melting data were obtained by monitoring $\mathrm{CD}$ signal at 208 and $222 \mathrm{~nm}$ while heating samples from $20^{\circ} \mathrm{C}$ to $94^{\circ} \mathrm{C}$ in a step size of $1^{\circ} \mathrm{C}$. A CD buffer spectrum was subtracted from each TOG domain spectrum, and the second derivative was taken for each melt curve to determine inflection points. All CD spectra were smoothed using Chirascan-plus software before analysis. CD data were completed on two separate experimental days with representative traces shown.

\section{Immunofluorescence microscopy, colocalization, and mitotic phenotype/index classification}

Cells were seeded onto coverslips treated with $0.5 \mathrm{mg} / \mathrm{ml}$ ConA and attached by UV-curable adhesive (Norland Products) to drilled $35-\mathrm{mm}$ tissue culture dishes in Schneider's medium supplemented with $10 \%$ FBS and $1 \times$ Anti-Anti. After $2 \mathrm{~h}$, cells were washed briefly with PBS and fixed using $-80^{\circ} \mathrm{C}$ precooled methanol for $8 \mathrm{~min}$ at $-20^{\circ} \mathrm{C}$. Fixed cells were washed $3 \times$ with PBST (PBS containing $0.1 \%$ Tween 20), allowed to block for $3 \mathrm{~h}$ at RT in blocking buffer (PBST supplemented with $0.2 \%$ BSA; Thermo Fisher Scientific), and incubated overnight at $4{ }^{\circ} \mathrm{C}$ with primary antibodies. Antibodies used in this study included guinea pig anti-Asterless (G. Rogers, University of Arizona Cancer Center, Tucson, AZ) 1:15,000; mouse monoclonal anti- $\alpha$ tubulin (DM1 $\alpha$, T6199; Sigma-Aldrich) 1:1,000, and rabbit polyclonal anti-phospho-histone H3 (06-570; EMD Millipore) 1:500. Cells were washed $3 \times$ with PBST, and secondary antibody was added for $1 \mathrm{~h}$ at $4^{\circ} \mathrm{C}$. Secondary antibodies included DAPI (Molecular Probes, D1306; Invitrogen) 1:1,000 and Cy2, Cy3, Cy5 (Jackson ImmunoResearch Laboratories, Inc.) 1:500. Secondary antibodies were removed and cells were washed $3 \times$ with PBST, and stored in PBS. Cells were imaged at RT with a Nikon Apo TIRF 100× oil/1.49-NA objective on an Eclipse Ti-E microscope (Nikon), driven by NIS Elements software (Nikon). Images were acquired with a CoolSNAP HQ cooled charge-coupled device camera (Roper Technologies).

For GST Linker4-TOG5 colocalization experiments, images were acquired of cells expressing GST Linker4-TOG5 constructs that were costained with anti- $\alpha$-tubulin. Strong, weak, and no binding were assigned. Strong binding was assigned if colocalization occurred throughout the cell analyzed, weak binding if there were only a few regions of colocalization, and no binding if the GST Linker4TOG5 construct was entirely cytoplasmic. At least 75 transfected cells were analyzed across three independent transfections. The percentage of cells exhibiting strong, weak, and no binding were plotted using a vertical stacked bar graph with the mean \pm SEM represented (Prism). For mitotic phenotype analysis, spindles were imaged using $z$-stacks, and four mitotic phenotype classes were scored manually as described previously (Goshima, 2010). In brief, bipolar and monastral bipolar mitotic spindles contained two poles with either two or one centrosome present, respectively. We characterized spindles as misaligned if the spindle MTs or DNA were not aligned properly, or if centrosomes were detached. Monopolar and multipolar mitotic spindles were classified as containing one or more than two centrosomes, respectively. Bipolar mitotic spindle pole-to-pole distance was measured as the distance between the two asterless signals using Fiji. Pole-to-pole distances were plotted using a bar graph with mean $\pm 95 \%$ confidence intervals represented and analyzed using a two-tailed nonparametric Mann-Whitney $U$ test (Prism). Images of Msps-eGFP constructs or tubulin (control and msps dsRNA-treated cells) and phospho-histone H3 were taken at random across each plate for mitotic index analysis. Cells in mitosis and interphase were manually counted, and the mitotic index was calculated and analyzed using two-way unpaired Student's $t$ tests. All images were analyzed using Fiji. Mitotic index and phenotypes were plotted in Prism displaying the mean \pm SEM.

\section{Statistical analyses}

To test whether data were normally distributed, a Lilliefors test was used. Normally distributed mitotic index data were analyzed using two-way unpaired Student's $t$ tests. Non-normally distributed data 
(EB1 comet velocities and pole-to-pole distances) were analyzed using a two-tailed nonparametric Mann-Whitney $U$ test. Unless otherwise noted, statistical significance was defined as $\mathrm{P}<0.05$.

\section{Online supplemental material}

Supplemental material includes the correlation of MT polymerization velocity with corrected mean cell fluorescence/pixel for all Msps-eGFP rescue constructs tracked using EB1-tRFP (Fig. S1); an alignment of TOG5 domains with $C e$ ZYG-9 TOG3 and CD data for Msps TOG5 HR 0 stability mutants (Fig. S2); MAPs modeled on the MT lattice (Fig. S3); and protein purification gels for all recombinant protein used in this study and CD data for native Msps TOG5 as well as HR 0-A mutants used in biochemical/cell experiments (Fig. S4).

\section{Acknowledgments}

We thank Matt Redinbo and laboratory for GM/CA-CAT assistance, Steve Rogers for reagents, the Advanced Photon Source and Ash Tripathy for technical assistance, Paul Maddox for experimental input, and Mark Peifer for manuscript comments.

This work was supported by the National Institutes of Health (T32GM008570 to the Program in Molecular and Cellular Biophysics at the University of North Carolina, Chapel Hill; R01GM094415 to K.C. Slep) and March of Dimes Foundation (FY1 1-434 to K.C. Slep). The National Institutes of Health (F31-GM115107) and American Heart Association (15PRE25380004) supported A.E. Byrnes.

The authors declare no competing financial interests.

Author contributions: A.E. Byrnes and K.C. Slep designed the research, analyzed and interpreted the data, and wrote the manuscript; A.E. Byrnes performed experiments.

Submitted: 25 October 2016

Revised: 2 March 2017

Accepted: 26 April 2017

\section{References}

Adams, P.D., P.V. Afonine, G. Bunkóczi, V.B. Chen, I.W. Davis, N. Echols, J.J.Headd, L.W. Huang, G.J. Kapral, R.W. Grosse-Kunstleve, et al. 2010. PHENIX: A comprehensive Python-based system for macromolecular structure solution. Acta Cryst. 66:213-221. http://dx.doi.org/10.1107/S0907444909052925

Akhmanova, A., C.C. Hoogenraad, K. Drabek, T. Stepanova, B. Dortland, T. Verkerk, W. Vermeulen, B.M. Burgering, C.I. De Zeeuw, F. Grosveld, and N. Galjart. 2001. Clasps are CLIP-115 and -170 associating proteins involved in the regional regulation of microtubule dynamics in motile fibroblasts. Cell. 104:923-935. http://dx.doi.org/10.1016/S0092-8674(01)00288-4

Al-Bassam, J., and F. Chang. 2011. Regulation of microtubule dynamics by TOG-domain proteins XMAP215/Dis1 and CLASP. Trends Cell Biol. 21:604-614. http://dx.doi.org/10.1016/j.tcb.2011.06.007

Al-Bassam, J., M. van Breugel, S.C. Harrison, and A. Hyman. 2006. Stu2p binds tubulin and undergoes an open-to-closed conformational change. J. Cell Biol. 172:1009-1022. http://dx.doi.org/10.1083/jcb.200511010

Al-Bassam, J., N.A. Larsen, A.A. Hyman, and S.C. Harrison. 2007. Crystal structure of a TOG domain: Conserved features of XMAP215/Dis1family TOG domains and implications for tubulin binding. Structure. 15:355-362. http://dx.doi.org/10.1016/j.str.2007.01.012

Al-Bassam, J., H. Kim, G. Brouhard, A. van Oijen, S.C. Harrison, and F. Chang. 2010. CLASP promotes microtubule rescue by recruiting tubulin dimers to the microtubule. Dev. Cell. 19:245-258. http://dx.doi.org/10.1016/j .devcel.2010.07.016

Al-Bassam, J., H. Kim, I. Flor-Parra, N. Lal, H. Velji, and F. Chang. 2012. Fission yeast Alp14 is a dose-dependent plus end-tracking microtubule polymerase. Mol. Biol. Cell. 23:2878-2890. http://dx.doi.org/10.1091/ mbc.E12-03-0205

Ayaz, P., X. Ye, P. Huddleston, C.A. Brautigam, and L.M. Rice. 2012. A TOG: $\alpha \beta-$ tubulin complex structure reveals conformation-based mechanisms for a microtubule polymerase. Science. 337:857-860. http://dx.doi.org/10 $.1126 /$ science. 1221698
Ayaz, P., S. Munyoki, E.A. Geyer, F.A. Piedra, E.S. Vu, R. Bromberg, Z. Otwinowski, N.V. Grishin, C.A. Brautigam, and L.M. Rice. 2014. A tethered delivery mechanism explains the catalytic action of a microtubule polymerase. eLife. 3:e03069. http://dx.doi.org/10.7554/eLife.03069

Baker, N.A., D. Sept, S. Joseph, M.J. Holst, and J.A. McCammon. 2001. Electrostatics of nanosystems: Application to microtubules and the ribosome. Proc. Natl. Acad. Sci. USA. 98:10037-10041. http://dx.doi.org /10.1073/pnas.181342398

Barr, A.R., and F. Gergely. 2008. MCAK-independent functions of ch-Tog/ XMAP215 in microtubule plus-end dynamics. Mol. Cell. Biol. 28:71997211. http://dx.doi.org/10.1128/MCB.01040-08

Bratman, S.V., and F. Chang. 2007. Stabilization of overlapping microtubules by fission yeast CLASP. Dev. Cell. 13:812-827. http://dx.doi.org/10.1016/j .devcel.2007.10.015

Brouhard, G.J., and L.M. Rice. 2014. The contribution of $\alpha \beta$-tubulin curvature to microtubule dynamics. J. Cell Biol. 207:323-334. http://dx.doi.org/10 .1083/jcb.201407095

Brouhard, G.J., J.H. Stear, T.L. Noetzel, J. Al-Bassam, K. Kinoshita, S.C. Harrison, J. Howard, and A.A. Hyman. 2008. XMAP215 is a processive microtubule polymerase. Cell. 132:79-88. http://dx.doi.org /10.1016/j.cell.2007.11.043

Cassimeris, L., D. Gard, P.T. Tran, and H.P. Erickson. 2001. XMAP215 is a long thin molecule that does not increase microtubule stiffness. J. Cell Sci. 114:3025-3033. http://dx.doi.org/10.1002/cm.20359

Cassimeris, L., J. Morabito, P.T. Tran, and H.P. Erickson. 2004. TOGp, the human homolog of XMAP215/Dis1, is required for centrosome integrity, spindle pole organization, and bipolar spindle assembly. Mol. Biol. Cell. 15:1580-1590. http://dx.doi.org/10.1091/mbc.E03-07-0544

Cassimeris, L., B. Becker, and B. Carney. 2009. TOGp regulates microtubule assembly and density during mitosis and contributes to chromosome directional instability. Cell Motil. Cytoskeleton. 66:535-545. http://dx .doi.org/10.1002/cm.20359

Cullen, C.F., P. Deák, D.M. Glover, and H. Ohkura. 1999. Mini spindles: A gene encoding a conserved microtubule-associated protein required for the integrity of the mitotic spindle in Drosophila. J. Cell Biol. 146:10051018. http://dx.doi.org/10.1083/jcb.146.5.1005

Currie, J.D., S. Stewman, G. Schimizzi, K.C. Slep, A. Ma, and S.L. Rogers. 2011. The microtubule lattice and plus-end association of Drosophila Mini spindles is spatially regulated to fine-tune microtubule dynamics. Mol. Biol. Cell. 22:4343-4361. http://dx.doi.org/10.1091/mbc.E11-06-0520

Das, A., D.J. Dickinson, C.C. Wood, B. Goldstein, and K.C. Slep. 2015. Crescerin uses a TOG domain array to regulate microtubules in the primary cilium. Mol. Biol. Cell. 26:4248-4264. http://dx.doi.org/10.1091 /mbc.E15-08-0603

De la Mora-Rey, T., B.D. Guenther, and B.C. Finzel. 2013. The structure of the TOG-like domain of Drosophila melanogaster Mast/Orbit. Acto Crystallogr. Sect. F Struct. Biol. Cryst. Commun. 69:723-729. http://dx .doi.org/10.1107/S1744309113015182

De Wulf, P., A.D. McAinsh, and P.K. Sorger. 2003. Hierarchical assembly of the budding yeast kinetochore from multiple subcomplexes. Genes Dev. 17:2902-2921. http://dx.doi.org/10.1101/gad.1144403

Emsley, P., B. Lohkamp, W.G. Scott, and K. Cowtan. 2010. Features and development of Coot. Acta Cryst. 66:486-501. http://dx.doi.org/10.1107 /S0907444910007493

Fox, J.C., A.E. Howard, J.D. Currie, S.L. Rogers, and K.C. Slep. 2014. The XMAP215 family drives microtubule polymerization using a structurally diverse TOG array. Mol. Biol. Cell. 25:2375-2392. http://dx.doi.org/10 .1091/mbc.E13-08-0501

Garcia, M.A., L. Vardy, N. Koonrugsa, and T. Toda. 2001. Fission yeast chTOG/XMAP215 homologue Alp14 connects mitotic spindles with the kinetochore and is a component of the Mad2-dependent spindle checkpoint. EMBO J. 20:3389-3401. http://dx.doi.org/10.1093/emboj/20 .13 .3389

Garcia, M.A., N. Koonrugsa, and T. Toda. 2002. Spindle-kinetochore attachment requires the combined action of Kin I-like Klp5/6 and Alp14/Dis1-MAPs in fission yeast. EMBO J. 21:6015-6024. http://dx.doi.org/10.1093/ emboj/cdf611

Gard, D.L., and M.W. Kirschner. 1987. A microtubule-associated protein from Xenopus eggs that specifically promotes assembly at the plus-end. J. Cell Biol. 105:2203-2215. http://dx.doi.org/10.1083/jcb.105.5.2203

Goshima, G. 2010. Assessment of mitotic spindle phenotypes in Drosophila S2 cells. Methods Cell Biol. 97:259-275. http://dx.doi.org/10.1016/S0091 $-679 X(10) 97015-5$

Goshima, G., and R.D. Vale. 2005. Cell cycle-dependent dynamics and regulation of mitotic kinesins in Drosophila S2 cells. Mol. Biol. Cell. 16:3896-3907. http://dx.doi.org/10.1091/mbc.E05-02-0118 
Goshima, G., R. Wollman, N. Stuurman, J.M. Scholey, and R.D. Vale. 2005. Length control of the metaphase spindle. Curr. Biol. 15:1979-1988. http ://dx.doi.org/10.1016/j.cub.2005.09.054

Grode, K.D., and S.L. Rogers. 2015. The non-catalytic domains of Drosophila katanin regulate its abundance and microtubule-disassembly activity. PLoS One. 10:e0123912. http://dx.doi.org/10.1371/journal.pone.0123912

Guesdon, A., F. Bazile, R.M. Buey, R. Mohan, S. Monier, R.R. García, M. Angevin, C. Heichette, R. Wieneke, R. Tampé, et al. 2016. EB1 interacts with outwardly curved and straight regions of the microtubule lattice. Nat. Cell Biol. 18:1102-1108. http://dx.doi.org/10.1038/ ncb3412

Hasegawa, H., and L. Holm. 2009. Advances and pitfalls of protein structural alignment. Curr. Opin. Struct. Biol. 19:341-348. http://dx.doi.org/10 .1016/j.sbi.2009.04.003

Heck, M.M., A. Pereira, P. Pesavento, Y. Yannoni, A.C. Spradling, and L.S. Goldstein. 1993. The kinesin-like protein KLP61F is essential for mitosis in Drosophila. J. Cell Biol. 123:665-679. http://dx.doi.org/10 $.1083 /$ jcb.123.3.665

Holmfeldt, P., S. Stenmark, and M. Gullberg. 2004. Differential functional interplay of TOGp/XMAP215 and the KinI kinesin MCAK during interphase and mitosis. EMBO J. 23:627-637. http://dx.doi.org/10.1038 /sj.emboj.7600076

Howard, A.E., J.C. Fox, and K.C. Slep. 2015. Drosophila melanogaster mini spindles TOG3 utilizes unique structural elements to promote domain stability and maintain a TOG1- and TOG2-like tubulin-binding surface. J. Biol. Chem. 290:10149-10162. http://dx.doi.org/10.1074/jbc.M114.633826

Hsu, K.S., and T. Toda. 2011. Ndc80 internal loop interacts with Dis1/TOG to ensure proper kinetochore-spindle attachment in fission yeast. Curr. Biol. 21:214-220. http://dx.doi.org/10.1016/j.cub.2010.12.048

Kabsch, W. 2010. XDS. Acta Crystallogr. D Biol. Crystallogr. 66:125-132. http ://dx.doi.org/10.1107/S0907444909047337

Kerssemakers, J.W., E.L. Munteanu, L. Laan, T.L. Noetzel, M.E. Janson, and M. Dogterom. 2006. Assembly dynamics of microtubules at molecular resolution. Nature. 442:709-712. http://dx.doi.org/10.1038/ nature 04928

Kosco, K.A., C.G. Pearson, P.S. Maddox, P.J. Wang, I.R. Adams, E.D. Salmon, K. Bloom, and T.C. Huffaker. 2001. Control of microtubule dynamics by Stu2p is essential for spindle orientation and metaphase chromosome alignment in yeast. Mol. Biol. Cell. 12:2870-2880. http://dx.doi.org/10 $.1091 / \mathrm{mbc} .12 .9 .2870$

Leano, J.B., S.L. Rogers, and K.C. Slep. 2013. A cryptic TOG domain with a distinct architecture underlies CLASP-dependent bipolar spindle formation. Structure. 21:939-950. http://dx.doi.org/10.1016/j.str.2013 .04 .018

Li, W., T. Moriwaki, T. Tani, T. Watanabe, K. Kaibuchi, and G. Goshima. 2012. Reconstitution of dynamic microtubules with Drosophila XMAP215, EB1, and Sentin. J. Cell Biol. 199:849-862. http://dx.doi.org/10.1083/ jcb.201206101

Liu, J.S., C.R. Schubert, X. Fu, F.J. Fourniol, J.K. Jaiswal, A. Houdusse, C.M. Stultz, C.A. Moores, and C.A. Walsh. 2012. Molecular basis for specific regulation of neuronal kinesin-3 motors by doublecortin family proteins. Mol. Cell. 47:707-721. http://dx.doi.org/10.1016/j.molcel.2012.06.025

Löwe, J., H. Li, K.H. Downing, and E. Nogales. 2001. Refined structure of $\alpha$ $\beta$-tubulin at 3.5 A resolution. J. Mol. Biol. 313:1045-1057. http://dx.doi .org/10.1006/jmbi.2001.5077

Maki, T., A.D. Grimaldi, S. Fuchigami, I. Kaverina, and I. Hayashi. 2015. CLASP2 has two distinct TOG domains that contribute differently to microtubule dynamics. J. Mol. Biol. 427:2379-2395. http://dx.doi.org/10 .1016/j.jmb.2015.05.012

Matthews, L.R., P. Carter, D. Thierry-Mieg, and K. Kemphues. 1998. ZYG-9, a Caenorhabditis elegans protein required for microtubule organization and function, is a component of meiotic and mitotic spindle poles. J. Cell Biol. 141:1159-1168. http://dx.doi.org/10.1083/jcb.141.5.1159

Maurer, S.P., P. Bieling, J. Cope, A. Hoenger, and T. Surrey. 2011. GTP $\gamma$ S microtubules mimic the growing microtubule end structure recognized by end-binding proteins (EBs). Proc. Natl. Acad. Sci. USA. 108:3988-3993. http://dx.doi.org/10.1073/pnas.1014758108

Maurer, S.P., F.J. Fourniol, G. Bohner, C.A. Moores, and T. Surrey. 2012. EBs recognize a nucleotide-dependent structural cap at growing microtubule ends. Cell. 149:371-382. http://dx.doi.org/10.1016/j.cell.2012.02.049

Maurer, S.P., N.I. Cade, G. Bohner, N. Gustafsson, E. Boutant, and T. Surrey. 2014. EB1 accelerates two conformational transitions important for microtubule maturation and dynamics. Curr. Biol. 24:372-384. http://dx .doi.org/10.1016/j.cub.2013.12.042

Meijering, E., O. Dzyubachyk, and I. Smal. 2012. Methods for cell and particle tracking. Methods Enzymol. 504:183-200. http://dx.doi.org/10.1016/ B978-0-12-391857-4.00009-4
Miller, M.P., C.L. Asbury, and S. Biggins. 2016. A TOG protein confers tension sensitivity to kinetochore-microtubule attachments. Cell. 165:14281439. http://dx.doi.org/10.1016/j.cell.2016.04.030

Mitchison, T., and M. Kirschner. 1984. Dynamic instability of microtubule growth. Nature. 312:237-242. http://dx.doi.org/10.1038/312237a0

Nakamura, S., I. Grigoriev, T. Nogi, T. Hamaji, L. Cassimeris, and Y. MimoriKiyosue. 2012. Dissecting the nanoscale distributions and functions of microtubule-end-binding proteins EB1 and ch-TOG in interphase $\mathrm{HeLa}$ cells. PLoS One. 7:e51442. http://dx.doi.org/10.1371/journal.pone.0051442

Nogales, E., S.G. Wolf, and K.H. Downing. 1998. Structure of the $\alpha \beta$ tubulin dimer by electron crystallography. Nature. 391:199-203. http://dx.doi .org/10.1038/34465

Popov, A.V., A. Pozniakovsky, I. Arnal, C. Antony, A.J. Ashford, K. Kinoshita, R. Tournebize, A.A. Hyman, and E. Karsenti. 2001. XMAP215 regulates microtubule dynamics through two distinct domains. EMBO J. 20:397410. http://dx.doi.org/10.1093/emboj/20.3.397

Popov, A.V., F. Severin, and E. Karsenti. 2002. XMAP215 is required for the microtubule-nucleating activity of centrosomes. Curr. Biol. 12:13261330. http://dx.doi.org/10.1016/S0960-9822(02)01033-3

Reber, S.B., J. Baumgart, P.O. Widlund, A. Pozniakovsky, J. Howard, A.A. Hyman, and F. Jülicher. 2013. XMAP215 activity sets spindle length by controlling the total mass of spindle microtubules. Nat. Cell Biol. 15:1116-1122. http://dx.doi.org/10.1038/ncb2834

Rice, L.M., E.A. Montabana, and D.A. Agard. 2008. The lattice as allosteric effector: Structural studies of $\alpha \beta$ - and $\gamma$-tubulin clarify the role of GTP in microtubule assembly. Proc. Natl. Acad. Sci. USA. 105:5378-5383. http ://dx.doi.org/10.1073/pnas.0801155105

Rizk, R.S., K.A. Discipio, K.G. Proudfoot, and M.L. Gupta Jr. 2014. The kinesin-8 Kip3 scales anaphase spindle length by suppression of midzone microtubule polymerization. J. Cell Biol. 204:965-975. http://dx.doi.org /10.1083/jcb.201312039

Rogers, S.L., and G.C. Rogers. 2008. Culture of Drosophila S2 cells and their use for RNAi-mediated loss-of-function studies and immunofluorescence microscopy. Nat. Protoc. 3:606-611. http://dx.doi.org/10.1038/nprot .2008 .18

Rogers, S.L., G.C. Rogers, D.J. Sharp, and R.D. Vale. 2002. Drosophila EB1 is important for proper assembly, dynamics, and positioning of the mitotic spindle. J. Cell Biol. 158:873-884. http://dx.doi.org/10.1083/jcb.200202032

Slep, K.C., and R.D. Vale. 2007. Structural basis of microtubule plus end tracking by XMAP215, CLIP-170, and EB1. Mol. Cell. 27:976-991. http ://dx.doi.org/10.1016/j.molcel.2007.07.023

Spittle, C., S. Charrasse, C. Larroque, and L. Cassimeris. 2000. The interaction of TOGp with microtubules and tubulin. J. Biol. Chem. 275:20748-20753. http://dx.doi.org/10.1074/jbc.M002597200

Thompson, J.D., D.G. Higgins, and T.J. Gibson. 1994. CLUSTAL W: Improving the sensitivity of progressive multiple sequence alignment through sequence weighting, position-specific gap penalties and weight matrix choice. Nucleic Acids Res. 22:4673-4680. http://dx.doi.org/10.1093/nar $/ 22.22 .4673$

Tournebize, R., A. Popov, K. Kinoshita, A.J. Ashford, S. Rybina, A. Pozniakovsky, T.U. Mayer, C.E. Walczak, E. Karsenti, and A.A. Hyman. 2000. Control of microtubule dynamics by the antagonistic activities of XMAP215 and XKCM1 in Xenopus egg extracts. Nat. Cell Biol. 2:13-19.

Trogden, K.P., and S.L. Rogers. 2015. TOG proteins are spatially regulated by Rac-GSK3 $\beta$ to control interphase microtubule dynamics. PLoS One. 10:e138966. http://dx.doi.org/10.1371/journal.pone.0138966

van Breugel, M., D. Drechsel, and A. Hyman. 2003. Stu2p, the budding yeast member of the conserved Dis1/XMAP215 family of microtubuleassociated proteins is a plus end-binding microtubule destabilizer. J. Cell Biol. 161:359-369. http://dx.doi.org/10.1083/jcb.200211097

van der Vaart, B., C. Manatschal, I. Grigoriev, V. Olieric, S.M. Gouveia, S. Bjelic, J. Demmers, I. Vorobjev, C.C. Hoogenraad, M.O. Steinmetz, and A. Akhmanova. 2011. SLAIN2 links microtubule plus end-tracking proteins and controls microtubule growth in interphase. J. Cell Biol. 193:1083-1099. http://dx.doi.org/10.1083/jcb.201012179

Vasquez, R.J., D.L. Gard, and L. Cassimeris. 1994. XMAP from Xenopus eggs promotes rapid plus end assembly of microtubules and rapid microtubule polymer turnover. J. Cell Biol. 127:985-993. http://dx.doi.org/10.1083/ jcb.127.4.985

Widlund, P.O., J.H. Stear, A. Pozniakovsky, M. Zanic, S. Reber, G.J. Brouhard, A.A. Hyman, and J. Howard. 2011. XMAP215 polymerase activity is built by combining multiple tubulin-binding TOG domains and a basic lattice-binding region. Proc. Natl. Acad. Sci. USA. 108:2741-2746. http ://dx.doi.org/10.1073/pnas.1016498108

Wieczorek, M., S. Bechstedt, S. Chaaban, and G.J. Brouhard. 2015. Microtubuleassociated proteins control the kinetics of microtubule nucleation. Nat. Cell Biol. 17:907-916. http://dx.doi.org/10.1038/ncb3188 
Yasuhara, H., and Y. Oe. 2011. TMBP200, a XMAP215 homologue of tobacco BY-2 cells, has an essential role in plant mitosis. Protoplasma. 248:493502. http://dx.doi.org/10.1007/s00709-010-0189-6
Zanic, M., P.O. Widlund, A.A. Hyman, and J. Howard. 2013. Synergy between XMAP215 and EB1 increases microtubule growth rates to physiological levels. Nat. Cell Biol. 15:688-693. http://dx.doi.org/10.1038/ncb2744 
